\title{
Brønsted Acid-Catalyzed Cyanotritylation of Aldehydes by Trityl Isocyanide
}

\author{
Răzvan C. Cioc, Peter Schuckman, Hans D. Preschel, Tjøstil Vlaar, Eelco Ruijter and \\ Romano V. A. Orru* \\ Department of Chemistry \& Pharmaceutical Sciences and Amsterdam Institute for Molecules Medicines \\ and Systems (AIMMS), Vrije Universiteit Amsterdam, De Boelelaan 1108, 1081 HZ Amsterdam, the \\ Netherlands
}

Email: r.v.a.orru@vu.nl 


\section{Contents}

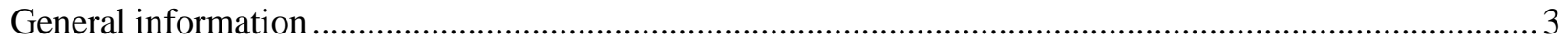

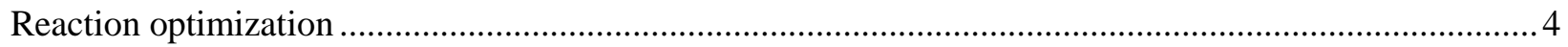

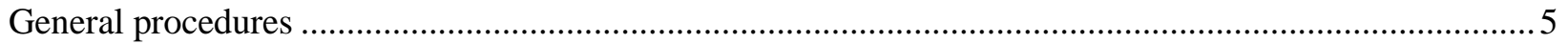

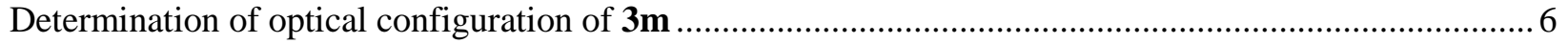

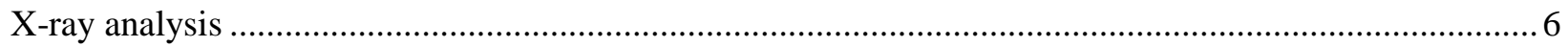

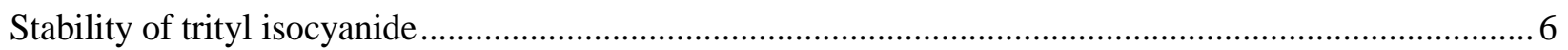

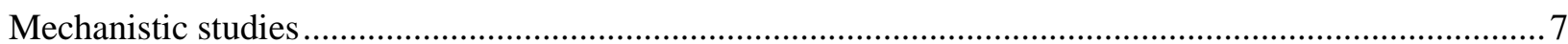

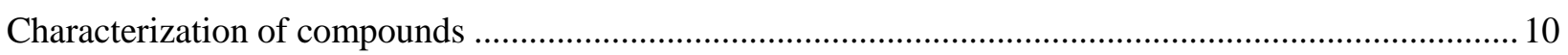

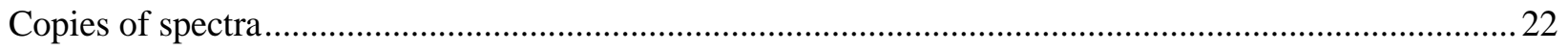

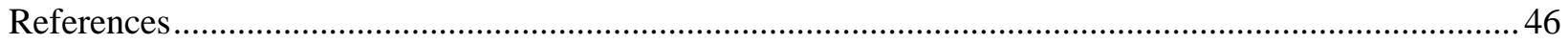




\section{General information}

Unless stated otherwise, all solvents and commercially available reagents were used as purchased. Cyclohexane was distilled prior to use. Dry toluene was distilled over sodium and stored under nitrogen on $4 \AA$ molecular sieves. All other solvents were used as purchased. All reactions were performed under nitrogen atmosphere.

Melting points were recorded on a Büchi M-565 melting point apparatus and are uncorrected. Nuclear magnetic resonance (NMR) spectra were recorded on a Bruker Avance $500\left(125.78 \mathrm{MHz}\right.$ for $\left.{ }^{13} \mathrm{C}\right)$ or Bruker Avance 400 (100.62 MHz for 13C) using the residual solvent as internal standard $\left({ }^{1} \mathrm{H}: \delta 7.26 \mathrm{ppm}\right.$, ${ }^{13} \mathrm{C}\left\{{ }^{1} \mathrm{H}\right\}: \delta 77.16 \mathrm{ppm}$ for $\mathrm{CDCl}_{3},{ }^{1} \mathrm{H}: \delta 2.50 \mathrm{ppm},{ }^{13} \mathrm{C}\left\{{ }^{1} \mathrm{H}\right\}: \delta 39.52 \mathrm{ppm}$ for DMSO-d6). Chemical shifts $(\delta)$ are given in ppm and coupling constants $(\mathrm{J})$ are quoted in hertz $(\mathrm{Hz})$. Resonances are described as $\mathrm{s}$ (singlet), d (doublet), t (triplet), q (quartet), quint (quintet), sex (sextet), sep (septet), br (broad singlet) and $\mathrm{m}$ (multiplet) or combinations thereof. Infrared (IR) spectra were recorded neat using a Shimadzu FTIR-8400s spectrophotometer and wavelengths are reported in $\mathrm{cm}^{-1}$. Electrospray Ionization (ESI) highresolution mass spectrometry (HRMS) was carried out using a Bruker microTOF-Q instrument in positive ion mode (capillary potential of $4500 \mathrm{~V}$ ). Flash chromatography was performed on Silicycle Silia-P Flash Silica Gel (particle size 40-63 $\mu \mathrm{m}$, pore diameter $60 \AA$ ) using the indicated eluent. Thin Layer Chromatography (TLC) was performed using TLC plates from Merck $\left(\mathrm{SiO}_{2}\right.$, Kieselgel 60 F254 neutral, on aluminium with fluorescence indicator). Chiral HPLC was recorded using a LC10VP with a SCL-10A VP system controller, LC-10AT VP liquid chromatograph, SPD-M10A VP diode array detector and CTO-10AC VP column oven from Shimadzu. X-ray analysis was performed on an Agilent SuperNova diffractometer with $\mathrm{Cu} \mathrm{K}(\alpha)$ microsource, mirror monochromator and Atlas $\mathrm{CCD}$ detector. The data were reduced and corrected for absorption with CrysAlisPro, Agilent Technologies, Version 1.171.37.35 (release 13-08-2014 CrysAlis171 .NET). The structure was solved with SHELXD (Sheldrick, 2008) and refined with SHELXL (Sheldrick, 2008) and the ShelxLE graphical interface (Hübschle, 2011).

Aldehydes $\mathbf{1 e}$ and $\mathbf{1 l}$ were prepared by oxidation of the corresponding alcohols. ${ }^{1}$ Spectra were in accordance with literature reports. ${ }^{2}$

Trityl isocyanide was synthesized as previously reported. ${ }^{3}$ 


\section{Reaction optimization}

To a solution of isovaleraldehyde $(0.25 \mathrm{mmol}, 1$ equiv) in toluene was added the diphenyl phosphoric acid catalyst ( $0.025 \mathrm{mmol}, 0.1$ equiv). Then, trityl isocyanide $(0.275 \mathrm{mmol}, 1.1$ equiv) was added and the resulted pale yellow solution was stirred at room temperature for 1-24 $\mathrm{h}$ (isocyanide consumption monitored with TLC). The reaction was then quenched with triethylamine ( $0.05 \mathrm{mmol}, 0.2$ equiv) and concentrated in vacuo. The crude yield was determined by NMR analysis using mesitylene as internal standard.

Table 1. Optimization of reaction conditions ${ }^{[a]}$

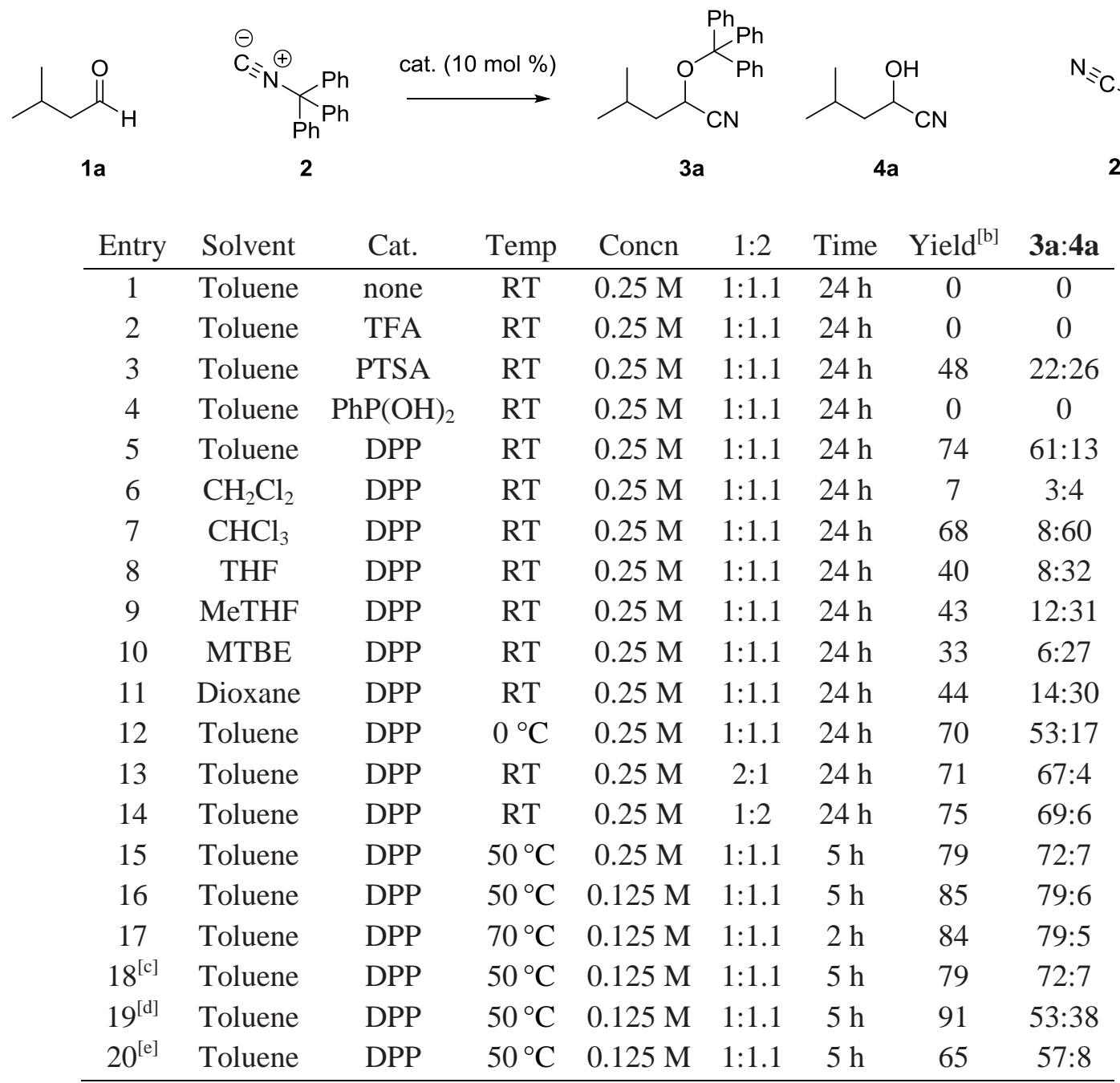

[a] Standard conditions: isovaleraldehyde $(0.25 \mathrm{mmol})$ in toluene, DPP $(10 \mathrm{~mol} \%)$ and trityl isocyanide (1.1 equiv) stirred at room temperature for the indicated time (isocyanide conversion monitored with TLC); [b] Combined yield of 3 and 4 based on NMR analysis with internal standard; [c] using freshly distilled toluene and inert conditions; [d] With addition of $3 \AA$ molecular sieves; [e] With addition of $\mathrm{MgSO}_{4}$. 


\section{General procedures}

\section{Procedure A. Synthesis of O-trityl cyanohydrins}

To a solution of aldehyde ( $0.5 \mathrm{mmol}, 1$ equiv) in toluene $(4 \mathrm{~mL})$ was added the diphenyl phosphoric acid catalyst ( $0.05 \mathrm{mmol}, 0.1$ equiv). Then, trityl isocyanide $(0.55 \mathrm{mmol}, 1.1$ equiv) was added and the resulted pale yellow solution was stirred at $50{ }^{\circ} \mathrm{C}$ for $5-24 \mathrm{~h}$ (isocyanide consumption monitored with TLC). The reaction was then quenched with triethylamine ( $0.1 \mathrm{mmol}, 0.2$ equiv) and concentrated in vacuo. The product was isolated by column chromatography on silicagel.

Note: in some instances the product co-elutes with impurities (generally triphenylacetonitrile). Gradient elution was employed (starting with cyclohexane/EtOAc 100/1).

Procedure B. One-pot synthesis of cyanohydrins

To a solution of aldehyde $(0.5 \mathrm{mmol}, 1$ equiv) in toluene $(4 \mathrm{~mL})$ was added the diphenyl phosphoric acid catalyst ( $0.05 \mathrm{mmol}, 0.1$ equiv). Then, trityl isocyanide $(0.55 \mathrm{mmol}, 1.1$ equiv) was added and the resulted pale yellow solution was stirred at $50{ }^{\circ} \mathrm{C}$ for $5-24 \mathrm{~h}$ (isocyanide consumption monitored with TLC). The reaction mixture was concentrated in vacuo and redissolved in trifluoroacetic acid (1 mL). Then, $\mathrm{Et}_{3} \mathrm{SiH}$ (0.75 mmol, 1.5 equiv) was added slowly. Upon addition, the intense yellow color disappeared within seconds and a white precipitate was formed. The mixture was stirred for $1 \mathrm{~h}$ at room temperature. The volatiles were removed and the product was isolated by column chromatography on silicagel.

\section{Procedure C. Passerini reaction}

The aldehyde $(0.5 \mathrm{mmol}, 1$ equiv), carboxylic acid $(0.5 \mathrm{mmol})$ and trityl isocyanide $(0.55 \mathrm{mmol}, 1.1$ equiv) were dissolved in dichloromethane $(1 \mathrm{~mL})$ and the solution was stirred at reflux for $24-48 \mathrm{~h}$ (isocyanide consumption monitored with TLC). The reaction was concentrated in vacuo and the product was isolated by column chromatography on silicagel.

\section{Procedure D. Deprotection of Passerini products}

The Passerini product ( $0.2 \mathrm{mmol}$. 1 equiv) was dissolved in a mixture of TFA-dichloromethane (4:1, 0.4 $\mathrm{mL})$. Then, triethylsilane was added $(0.3 \mathrm{mmol}, 1.5$ equiv) and the solution was stirred at room temperature for 1-20 h. The mixture was then quenched in saturated $\mathrm{NaHCO}_{3}$ solution $(10 \mathrm{~mL})$, and the product was extracted in dichloromethane (two times). Subsequently, the organic layers where combined and dried over $\mathrm{Na}_{2} \mathrm{SO}_{4}$. The solvents were removed in vacuo and the product was isolated by column chromatography on silicagel or trituration with pentane.

\section{Procedure E. Synthesis of enantioenriched O-trityl cyanohydrins}

To a solution of aldehyde $(0.25 \mathrm{mmol}, 1$ equiv) in toluene $(0.25 \mathrm{~mL})$ was added the $(R)$-TRIP $(19 \mathrm{mg}$, $0.025 \mathrm{mmol}, 0.1$ equiv). Then, trityl isocyanide $(0.275 \mathrm{mmol}, 1.1$ equiv) in toluene $(1 \mathrm{~mL})$ was added dropwise over $1 \mathrm{~h}$ and the resulted clear solution was stirred at room temperature for $48 \mathrm{~h}$. The reaction was then quenched with triethylamine $(0.05 \mathrm{mmol}, 0.2$ equiv) and concentrated in vacuo. The product was isolated by column chromatography on silicagel. The ee was determined by chiral HPLC analysis. 


\section{Determination of optical configuration of $3 \mathrm{~m}$}

Enantioenriched 3m (10 mg) was dissolved in dichloromethane-methanol (1:1, $0.4 \mathrm{~mL})$. Amberlyst® 15 (20 mg) was then added and the suspension was stirred at $60{ }^{\circ} \mathrm{C}$ for $4 \mathrm{~h}$. The mixture was cooled, Amberlyst was filtered off and the solvents were removed in vacuo. Chiral HPLC analysis of the crude reaction mixture indicated that the major enantiomer is the $S$ enantiomer (comparison with a commercial sample of $(R)-3 \mathbf{m})$. This result is consistent with the configuration established by X-ray analysis.

Chiral HPLC (Chiracel OD-H, heptane/isopropanol $=95 / 5, \mathrm{v}=1.0 \mathrm{~mL} / \mathrm{min}$, column temperature: $20{ }^{\circ} \mathrm{C}$, $\lambda=214 \mathrm{~nm}) \mathrm{t}(S)-3 \mathrm{~m}=19.6, \mathrm{t}(R)-3 \mathrm{~m}=20.6 \mathrm{~min}$.

Note: 3a and 3m appear to have a different order of elution of enantiomers in the chiral HPLC. It is difficult to determine whether this is due to a different interaction with the chiral stationary phase during the analysis or the selectivity of the reaction changes with the aliphatic/aromatic nature of the aldehyde (particularly since the ee obtained for 3a was moderate). Herein we assume the selectivity of the reaction with $1 \mathbf{a}$ and $1 \mathbf{m}$ to be consistent, i.e. (S)-enantiomer is the major one in both cases.

\section{X-ray analysis}

XRD-quality single crystals were obtained by slow evaporation of a $\mathrm{CDCl}_{3}$ solution. A single crystal of $3 \mathbf{m}$ was mounted on a kapton loop and placed in a 100.0(1)K cold nitrogen stream on the diffractometer. Via $\omega$ scans, 116883 reflections were collected, of which 4227 were unique. Rint was $8.8 \%$.

The enantiopure source material crystallized in the chiral space group $\mathrm{P} 2{ }_{1}$, and the resulting atomic distances and angles are unremarkable. The Flack parameter was determined using 1811 Friedel pair quotients to 0.04(12), confirming the absolute configuration to be $S$. In the crystal packing there is a nonclassical hydrogen bond with an unexpectedly short distance, between the hydrogen on the chiral carbon and the cyano nitrogen.

Further details of the crystal and refinement can be found in the CIF in the Supporting Information.

\section{Stability of trityl isocyanide}

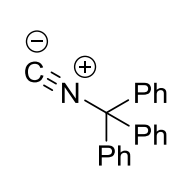

2

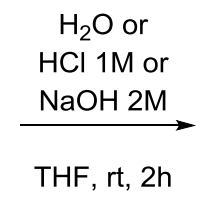

THF, rt, $2 \mathrm{~h}$

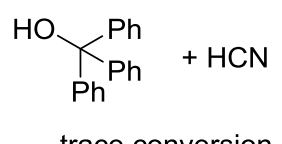

trace conversion

Trityl isocyanide $(27 \mathrm{mg}, 0.1 \mathrm{mmol})$ was dissolved in THF $(0.2 \mathrm{~mL})$. To this solution, water/HCl $1 \mathrm{M} / \mathrm{NaOH} 2 \mathrm{M}(0.2 \mathrm{~mL})$ was added. Upon mixing the isocyanide partly precipitated out. After $2 \mathrm{~h}$, ethyl acetate was added to dissolve all solids. TLC analysis indicated utmost traces of TrOH. 


\section{Mechanistic studies}

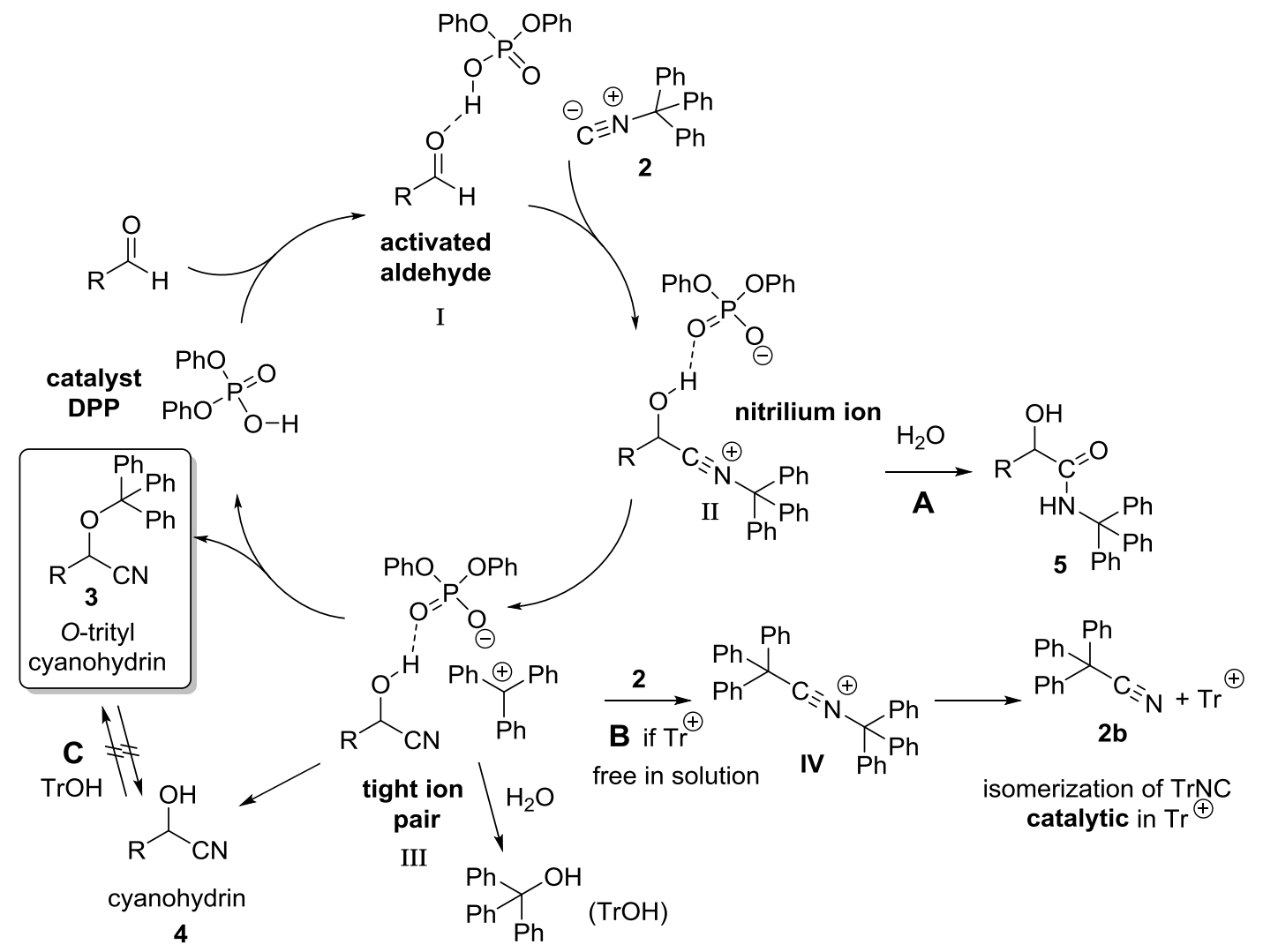

Scheme 1. Reaction mechanism

E1. Control experiment of trityl isocyanide stability in the reaction conditions

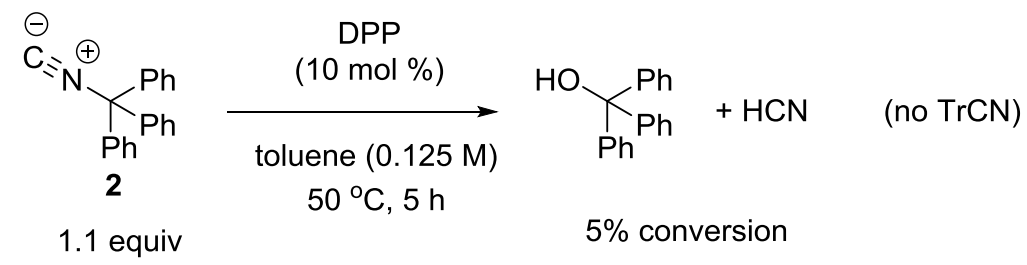

Trityl isocyanide (37 mg, $0.138 \mathrm{mmol}, 1.1$ equiv) was stirred in toluene ( $1 \mathrm{~mL})$ with DPP $(2.5 \mathrm{mg}, 0.01$ mmol, 0.1 equiv) at $50{ }^{\circ} \mathrm{C}$ for $5 \mathrm{~h}$. The reaction was then quenched with triethylamine $(14 \mu \mathrm{L}, 0.1 \mathrm{mmol}$, 1 equiv) and concentrated in vacuo. ${ }^{1} \mathrm{H}-\mathrm{NMR}$ and ${ }^{13} \mathrm{C}-\mathrm{NMR}$ analysis with mesitylene as internal standard indicated $5 \%$ conversion to $\mathrm{TrOH}$. TrCN was not observed.

Performing this experiment in dichloromethane showed only traces of $\mathrm{TrOH}$ but complete conversion of trityl isocyanide to $\mathrm{TrCN}$ within $1.5 \mathrm{~h}$ (TLC analysis). 
The release of HCN from trityl isocyanide is a slow process (in toluene) and cannot account for the conversions obtained in the tritylative cyanation reaction. In dichloromethane the initially formed DPP-Tr by loss of HCN induces the trityl isocyanide isomerization via the tritilium chain mechanism.

E2. Influence of base on the reaction

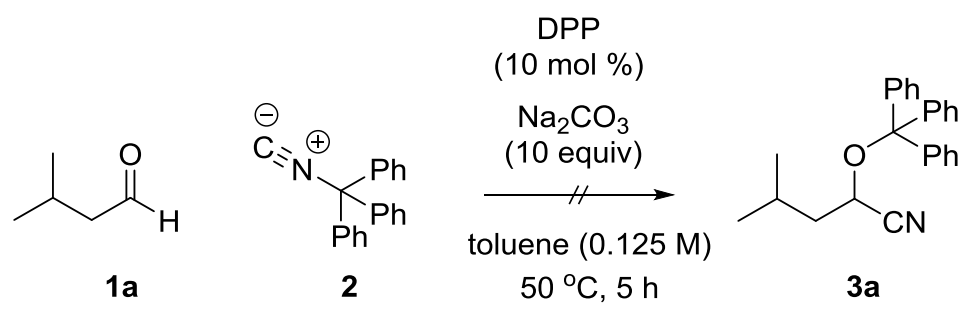

Prestirring DPP with $\mathrm{Na}_{2} \mathrm{CO}_{3}$ (10 equiv) under the optimal reaction conditions suppresses the reactivity completely (TLC analysis).

E3. Hydrolysis of 3a under the reaction conditions

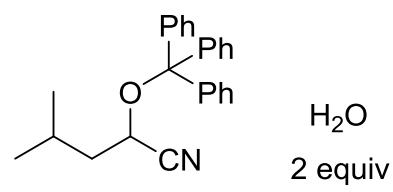

$3 \mathbf{a}$

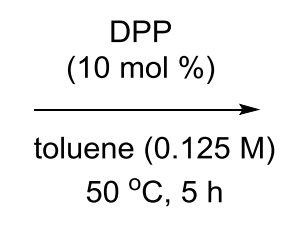

$50{ }^{\circ} \mathrm{C}, 5 \mathrm{~h}$<smiles>CC(C)CC(O)C#N</smiles>

$4 a$

$2 \%$ conversion

3a (71 mg, $0.2 \mathrm{mmol}, 1$ equiv) was stirred in toluene (1.6 mL) with DPP ( $5 \mathrm{mg}, 0.02 \mathrm{mmol}, 0.1$ equiv) and $\mathrm{H}_{2} \mathrm{O}\left(7.2 \mu \mathrm{L}, 0.4 \mathrm{mmol}, 2\right.$ equiv) at $50{ }^{\circ} \mathrm{C}$ for $5 \mathrm{~h}$. The reaction was then quenched with triethylamine (28 $\mu \mathrm{L}, 0.2 \mathrm{mmol}, 1$ equiv) and concentrated in vacuo. ${ }^{1} \mathrm{H}-\mathrm{NMR}$ analysis with mesitylene as internal standard indicated $2 \%$ conversion to $\mathbf{4 a}$ and $\mathrm{TrOH}$.

During the tritylative cyanation of $\mathbf{1 a}, \mathbf{4 a}$ is formed mostly by isocyanide addition to $\mathbf{1 a}$ and fragmentation of II. Formation of $\mathbf{4 a}$ by hydrolysis of $\mathbf{3 a}$ (pathway $\mathbf{C}$ ) is not favored kinetically.

E4. Reaction in the presence of water

Reaction was done according to Procedure A with the addition of 0.5 equiv $\mathrm{H}_{2} \mathrm{O}$. The reaction outcome was analyzed by NMR with mesitylene as internal standard.

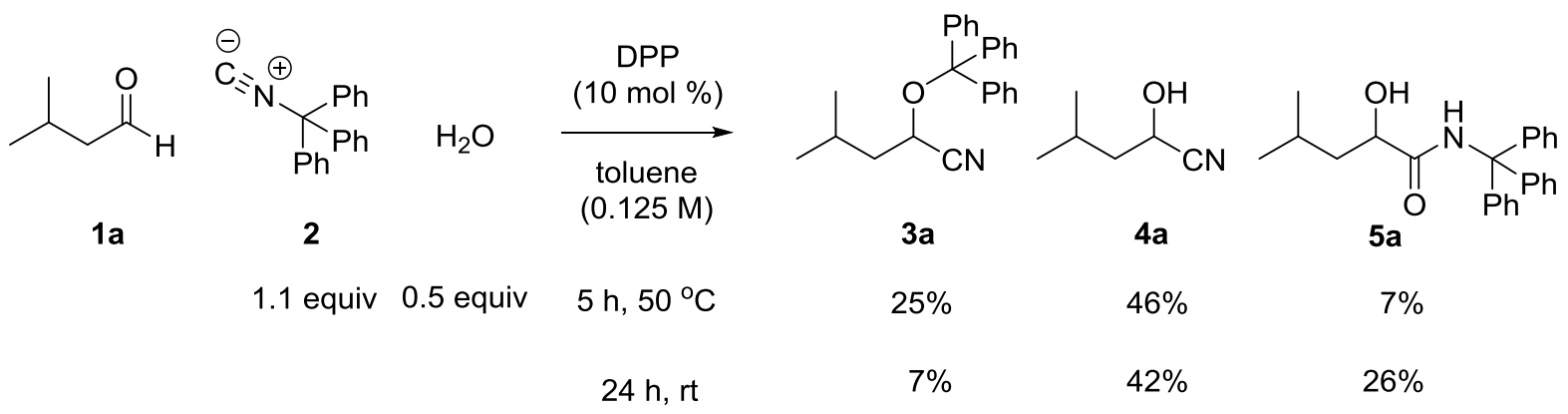


The reaction in the presence of water is slower $\left(\mathrm{H}_{2} \mathrm{O}\right.$ competes with 1a to hydrogen bond with DPP) and it does not go to completion within $5 \mathrm{~h}$ at $50{ }^{\circ} \mathrm{C}$. The selectivity is in favor of $4 \mathbf{a}$ over $3 \mathbf{a}$ (presumably water is also bound to DPP at stage II and III and traps the tritylium resulted by the fragmentation of the nitrilium ion (indeed, in such experiments the isomerization to $\operatorname{TrCN}$ was completely suppressed). A minor amount of 5a is also formed by the trapping of the nitrilium ion with water (stage II). When the reaction is performed at room temperature, the selectivity towards $\mathbf{5 a}$ increases to $35 \%$ compared to $9 \%$ at $50{ }^{\circ} \mathrm{C}$ (this allowed the isolation and characterization of 5a). We reason that the nitrilium ion has a longer lifetime at room temperature and water addition occurs at a comparable rate with the fragmentation whereas at higher temperatures the rate of fragmentation is greatly enhanced and only a minor amount of 5a is formed.

E5. Tritylation of $4 \mathbf{a}$ to $3 \mathbf{a}$ with $\mathrm{TrOH}$

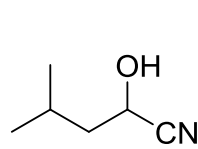

$4 \mathbf{a}$
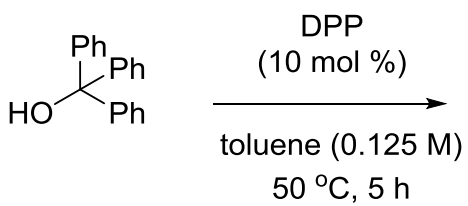

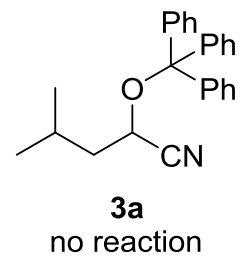

4a (11 mg, $0.1 \mathrm{mmol}, 1$ equiv) was stirred in toluene $(0.8 \mathrm{~mL})$ with DPP ( $2.5 \mathrm{mg}, 0.01 \mathrm{mmol}, 0.1$ equiv) and $\mathrm{TrOH}\left(26 \mathrm{mg}, 0.1 \mathrm{mmol}, 1\right.$ equiv) at $50{ }^{\circ} \mathrm{C}$ for $5 \mathrm{~h}$. TLC analysis indicated no reaction.

Formation of $\mathbf{3 a}$ by tritylation of $\mathbf{4 a}$ with $\mathrm{TrOH}$ (pathway $\mathbf{C}$ ) is not possible (thermodynamically). The formation of 3a does not have $\mathrm{TrOH}$ as intermediate. Once the tritylium is intercepted by water traces it does not take part in the reaction.

E6. Tritylation of $\mathbf{4 a}$ to $3 \mathbf{a}$ with DPP-Tr

Synthesis of DPP-Tr

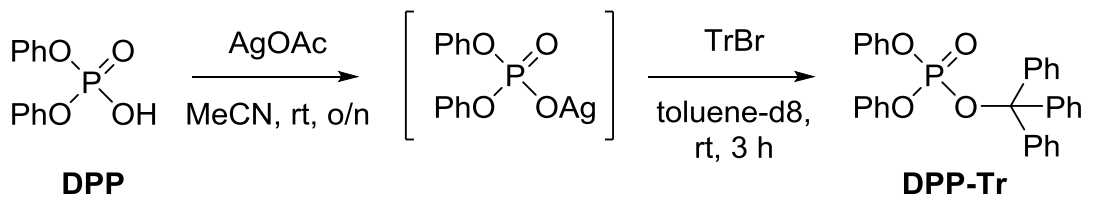

DPP (250 mg, $1 \mathrm{mmol}, 1.3$ equiv) and AgOAc (167 mg, $1 \mathrm{mmol}, 1.3$ equiv) were dissolved in anhydrous $\mathrm{MeCN}(2 \mathrm{~mL})$ under a nitrogen atmosphere. Upon stirring overnight, a white solid precipitated. The volatiles were removed in vacuo and the solid was suspended in toluene- $\mathrm{d} 8(2 \mathrm{~mL})$. Then, trityl bromide ( $242 \mathrm{mg}, 0.75 \mathrm{mmol}, 1$ equiv) was added portionwise. The mixture was stirred at room temperature for 3 $\mathrm{h}$ and then filtered. The filter was washed with toluene-d8 $(1 \mathrm{~mL}) .{ }^{31} \mathrm{P}-\mathrm{NMR}$ indicated complete conversion of DPP (-10 ppm) to DPP-Tr (-18 ppm); ${ }^{13} \mathrm{C}-\mathrm{NMR}$ analysis indicated complete conversion of TrBr. DPP-Tr was found hydrolytically unstable upon storage in toluene ( $\sim 50 \%$ hydrolysis to DPP and TrOH over 3 days). For this reason, DPP-Tr was not isolated but used as a toluene solution directly. 
Tritylation of $4 a$ with DPP-Tr
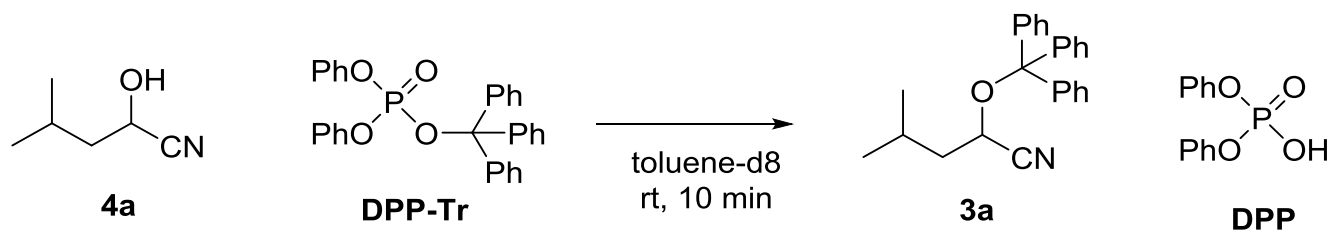

A solution of DPP-Tr $(0.1 \mathrm{mmol}, 1$ equiv) in toluene-d8 $(0.6 \mathrm{~mL})$ was added over a solution of $4 \mathbf{a}(0.1$ mmol, 1 equiv) in toluene-d8 $(0.2 \mathrm{~mL})$. Upon mixing of the two colorless solutions, an intense yellow color appeared which persisted for a few seconds. After $5 \mathrm{~min}$, the NMR of the solution was recorded.

${ }^{31} \mathrm{P}-\mathrm{NMR}$ indicated complete conversion of DPP-Tr to DPP; ${ }^{1} \mathrm{H}-\mathrm{NMR}$ indicated a $63 \%$ conversion of $4 \mathbf{a}$ to $\mathbf{3 a}$.

The trityl cation released upon isocyanide fragmentation (III) is rapidly trapped by neighboring nucleophiles. The reaction with $\mathbf{4}$ is fast, particularly since $\mathbf{4}$ is in the proximity (as it is in III), leading to observed product $\mathbf{3}$. If tritylium diffuses in solution (potentially as covalently bound to diphenylphosphate, DPP-Tr) it becomes available to other nucleophiles (water, trityl isocyanide).

\section{Characterization of compounds}<smiles>CC(C)CC(C#N)OC(c1ccccc1)(c1ccccc1)c1ccccc1</smiles>

\section{4-methyl-2-(trityloxy)pentanenitrile 3a}

Prepared from isovaleraldehyde (43 $\mathrm{mg}, 0.5 \mathrm{mmol}, 1$ equiv) and trityl isocyanide (148 $\mathrm{mg}, 0.55 \mathrm{mmol}$, 1.1 equiv) according to Procedure $\mathrm{A}(5 \mathrm{~h}$ reaction time). Purification: column chromatography on silicagel (cyclohexane/EtOAc 100/1, $\mathrm{R}_{\mathrm{f}}=0.30$ in cyclohexane/EtOAc 10:1). Isolated as a white solid. Yield: $122 \mathrm{mg}, 0.345 \mathrm{mmol}, 69 \%$ (co-elutes with triphenylacetonitrile, reported yield is corrected for 90 wt\% purity).

Large scale reaction: prepared from isovaleraldehyde $(517 \mathrm{mg}, 6.0 \mathrm{mmol}, 1$ equiv) and trityl isocyanide (1777 mg, $6.6 \mathrm{mmol}, 1.1$ equiv) according to Procedure A ( $5 \mathrm{~h}$ reaction time). Purification: column chromatography on silicagel (cyclohexane/EtOAc 100/1, $\mathrm{R}_{\mathrm{f}}=0.30$ in cyclohexane/EtOAc 10:1). Isolated as a white solid. Yield: $1258 \mathrm{mg}, 3.5 \mathrm{mmol}, 59 \%$ (co-elutes with triphenylacetonitrile, reported yield is corrected for $90 \mathrm{wt} \%$ purity).

m.p.: 71-85 ${ }^{\circ} \mathrm{C} ;{ }^{1} \mathrm{H}-\mathrm{NMR}\left(\mathrm{CDCl}_{3}, 500 \mathrm{MHz}\right): \delta$ 7.54-7.47 (m, 6H), 7.39-7.27 (m, 9H), $4.09(\mathrm{dd}, \mathrm{J}=8.5$, $6.0 \mathrm{~Hz}, 1 \mathrm{H}), 1.90-1.81(\mathrm{~m}, 1 \mathrm{H}), 1.80-1.73(\mathrm{~m}, 1 \mathrm{H}), 1.57-1.50(\mathrm{~m}, 1 \mathrm{H}), 0.85(\mathrm{~d}, \mathrm{~J}=6.6 \mathrm{~Hz}, 3 \mathrm{H}), 0.79(\mathrm{~d}, \mathrm{~J}$ $=6.3,3 \mathrm{H}) \mathrm{ppm} ;{ }^{13} \mathrm{C}\left\{{ }^{1} \mathrm{H}\right\}-\mathrm{NMR}\left(\mathrm{CDCl}_{3}, 125 \mathrm{MHz}\right): \delta 143.0(\mathrm{C}), 128.9(\mathrm{CH}), 128.3(\mathrm{CH}), 127.9(\mathrm{CH})$, $119.0(\mathrm{CN}), 89.1(\mathrm{C}), 62.2(\mathrm{CH}), 43.4\left(\mathrm{CH}_{2}\right), 24.4(\mathrm{CH}), 22.9\left(\mathrm{CH}_{3}\right), 22.1\left(\mathrm{CH}_{3}\right)$ ppm; IR (neat): vmax $\left(\mathrm{cm}^{-1}\right)=2957(\mathrm{~m}), 1489(\mathrm{~s}), 1448(\mathrm{~s}), 1221(\mathrm{~m}), 1059(\mathrm{~s}), 1032(\mathrm{~s}), 978(\mathrm{~m}), 899(\mathrm{~m}), 746(\mathrm{~s}), 696(\mathrm{~s})$, 630.7 (s); HRMS (ESI): $\mathrm{m} / z$ calculated for $\mathrm{C}_{25} \mathrm{H}_{25} \mathrm{NNaO}$ [M+Na] $]^{+} 378.1828$, found: 378.1823 . 
Enantioselective reaction: prepared from isovaleraldehyde (17 $\mathrm{mg}, 0.20 \mathrm{mmol}, 1$ equiv) and trityl isocyanide (60 $\mathrm{mg}, 0.22 \mathrm{mmol}, 1.1 \mathrm{equiv})$ according to Procedure E. Purification: column chromatography on silicagel (cyclohexane/EtOAc 15/1, $\mathrm{R}_{\mathrm{f}}=0.30$ in cyclohexane/EtOAc 10:1). Isolated as a white solid. Yield: $37 \mathrm{mg}, 0.104 \mathrm{mmol}, 52 \%$ (co-elutes with triphenylacetonitrile, reported yield is corrected for $90 \mathrm{wt} \%$ purity).

Chiral HPLC (Chiracel OD-H, heptane/isopropanol $=95 / 5, \mathrm{v}=0.5 \mathrm{~mL} / \mathrm{min}$, column temperature: $20{ }^{\circ} \mathrm{C}$, $\lambda=214 \mathrm{~nm}) \mathrm{t}($ minor $)-\mathbf{3 a}=9.3, \mathrm{t}($ major $)-\mathbf{3 a}=10.1 \mathrm{~min}, 55 \%$ ee.<smiles>CCCCCCC(C#N)OC(c1ccccc1)(c1ccccc1)c1ccccc1</smiles>

\section{2-(trityloxy)octanenitrile 3b}

Prepared from heptaldehyde (57 mg, $0.5 \mathrm{mmol}, 1$ equiv) and trityl isocyanide (148 mg, $0.55 \mathrm{mmol}, 1.1$ equiv) according to Procedure A ( $5 \mathrm{~h}$ reaction time). Purification: column chromatography on silicagel (cyclohexane/EtOAc 50/1, $\mathrm{R}_{\mathrm{f}}=0.58$ in cyclohexane/EtOAc 10:1). Isolated as a colorless oil. Yield: 117 $\mathrm{mg}, 0.305 \mathrm{mmol}, 61 \%$ (co-elutes with triphenylacetonitrile, reported yield is corrected for $92 \mathrm{wt} \%$ purity).

${ }^{1} \mathrm{H}-\mathrm{NMR}\left(\mathrm{CDCl}_{3}, 500 \mathrm{MHz}\right): \delta$ 7.58-7.51 (m, 6H), 7.43-7.36 (m, 6H), $7.33(\mathrm{tt}, \mathrm{J}=7.3,2.5 \mathrm{~Hz}, 3 \mathrm{H}), 4.17$ $(\mathrm{dd}, \mathrm{J}=7.6,4.7 \mathrm{~Hz}, 1 \mathrm{H}), 1.88-1.79(\mathrm{~m}, 1 \mathrm{H}), 1.74-1.66(\mathrm{~m}, 1 \mathrm{H}), 1.66-1.56(\mathrm{~m}, 1 \mathrm{H}), 1.50-1.39(\mathrm{~m}, 1 \mathrm{H})$, 1.38-1.24 (m, 6H), $0.93(\mathrm{t}, \mathrm{J}=6.9 \mathrm{~Hz}, 3 \mathrm{H}) \mathrm{ppm} ;{ }^{13} \mathrm{C}\left\{{ }^{1} \mathrm{H}\right\}-\mathrm{NMR}\left(\mathrm{CDCl}_{3}, 125 \mathrm{MHz}\right): \delta 143.0(\mathrm{C}), 128.8$ $(\mathrm{CH}), 128.2(\mathrm{CH}), 127.9(\mathrm{CH}), 118.7(\mathrm{CN}), 88.9(\mathrm{C}), 63.3(\mathrm{CH}), 34.6\left(\mathrm{CH}_{2}\right), 31.6\left(\mathrm{CH}_{2}\right), 28.8\left(\mathrm{CH}_{2}\right), 24.4$ $\left(\mathrm{CH}_{2}\right), 22.6\left(\mathrm{CH}_{2}\right), 14.1\left(\mathrm{CH}_{3}\right)$ ppm; IR (neat): vmax $\left(\mathrm{cm}^{-1}\right)=2955(\mathrm{~m}), 2930(\mathrm{~m}), 1489(\mathrm{~s}), 1447(\mathrm{~m})$, 1221 (m), 1061 (s), 1032 (s), 899 (m), 748 (s), 696 (s), 644 (s); HRMS (ESI): m/z calculated for $\mathrm{C}_{27} \mathrm{H}_{29} \mathrm{NNaO}[\mathrm{M}+\mathrm{Na}]^{+}$406.2141, found: 406.2137 .<smiles>N#CC(COCc1ccccc1)OC(c1ccccc1)(c1ccccc1)c1ccccc1</smiles>

\section{3-(benzyloxy)-2-(trityloxy)propanenitrile 3c}

Prepared from (benzyloxy)acetaldehyde (75 mg, $0.5 \mathrm{mmol}, 1$ equiv) and trityl isocyanide (148 $\mathrm{mg}, 0.55$ mmol, 1.1 equiv) according to Procedure A ( $5 \mathrm{~h}$ reaction time). Purification: column chromatography on silicagel (cyclohexane/EtOAc 100/1, $\mathrm{R}_{\mathrm{f}}=0.31$ in cyclohexane/EtOAc 10:1). Isolated as a colorless oil. Yield: $136 \mathrm{mg}, 0.325 \mathrm{mmol}, 65 \%$ (co-elutes with triphenylacetonitrile, reported yield is corrected for 96 wt $\%$ purity).

${ }^{1} \mathrm{H}-\mathrm{NMR}\left(\mathrm{CDCl}_{3}, 500 \mathrm{MHz}\right): \delta$ 7.58-7.51 (m, 6H), 7.43-7.30 (m, 14H), $4.62(\mathrm{~d}, \mathrm{~J}=12.0 \mathrm{~Hz}, 1 \mathrm{H}), 4.57(\mathrm{~d}$, $\mathrm{J}=12.0 \mathrm{~Hz}, 1 \mathrm{H}), 4.37(\mathrm{t}, \mathrm{J}=6.0 \mathrm{~Hz}, 1 \mathrm{H}), 3.71(\mathrm{dd}, \mathrm{J}=10.4,6.3 \mathrm{~Hz}, 1 \mathrm{H}), 3.62(\mathrm{dd}, \mathrm{J}=10.4,6.0 \mathrm{~Hz}, 1 \mathrm{H})$ ppm; ${ }^{13} \mathrm{C}\left\{{ }^{1} \mathrm{H}\right\}$-NMR $\left(\mathrm{CDCl}_{3}, 125 \mathrm{MHz}\right): \delta 142.6(\mathrm{C}), 137.3(\mathrm{C}), 128.9(\mathrm{CH}), 128.6(\mathrm{CH}), 128.3(\mathrm{CH})$, $128.1(\mathrm{CH}), 128.0(\mathrm{CH}), 127.8(\mathrm{CH}), 117.4(\mathrm{CN}), 89.3(\mathrm{C}), 73.7\left(\mathrm{CH}_{2}\right), 70.5\left(\mathrm{CH}_{2}\right), 63.0(\mathrm{CH}) \mathrm{ppm}$; IR 
(neat): vmax $\left(\mathrm{cm}^{-1}\right)=3059(\mathrm{~m}), 2966(\mathrm{~m}), 2177(\mathrm{~s}), 1489(\mathrm{~s}), 1447(\mathrm{~s}), 1250(\mathrm{~s}), 1084(\mathrm{~s}), 837(\mathrm{~s}), 750(\mathrm{~s})$, 706 (s), 633 (s); HRMS (ESI): $\mathrm{m} / z$ calculated for $\mathrm{C}_{29} \mathrm{H}_{25} \mathrm{NNaO}_{2}[\mathrm{M}+\mathrm{Na}]^{+}$442.1778, found: 442.1773 .<smiles>N#CC(Cc1ccccc1)OC(c1ccccc1)(c1ccccc1)c1ccccc1</smiles>

\section{3-phenyl-2-(trityloxy)propanenitrile 3d}

Prepared from 2-phenylacetaldehyde (60 mg, $0.5 \mathrm{mmol}, 1$ equiv) and trityl isocyanide (148 $\mathrm{mg}, 0.55$ mmol, 1.1 equiv) according to Procedure A ( $5 \mathrm{~h}$ reaction time). Purification: column chromatography on silicagel (cyclohexane/EtOAc 100/1, $\mathrm{R}_{\mathrm{f}}=0.39$ in cyclohexane/EtOAc 10:1). Isolated as a white solid. Yield: $82 \mathrm{mg}, 0.210 \mathrm{mmol}, 42 \%$ (co-elutes with triphenylacetonitrile, reported yield is corrected for 98 wt\% purity).

m.p.: 135-139 ${ }^{\circ} \mathrm{C} ;{ }^{1} \mathrm{H}-\mathrm{NMR}\left(\mathrm{CDCl}_{3}, 500 \mathrm{MHz}\right): \delta$ 7.53-7.45 (m, 6H), 7.39-7.27 (m, 12H), $7.18(\mathrm{dd}, \mathrm{J}=$ 7.9, $1.6 \mathrm{~Hz}, 2 \mathrm{H}), 4.32(\mathrm{dd}, \mathrm{J}=7.9,5.7 \mathrm{~Hz}, 1 \mathrm{H}), 3.05(\mathrm{dd}, \mathrm{J}=13.6,7.9 \mathrm{~Hz}, 1 \mathrm{H}), 2.85(\mathrm{dd}, \mathrm{J}=13.6,5.4 \mathrm{~Hz}$, 1H) ppm; ${ }^{13} \mathrm{C}\left\{{ }^{1} \mathrm{H}\right\}-\mathrm{NMR}\left(\mathrm{CDCl}_{3}, 125 \mathrm{MHz}\right): \delta 142.8(\mathrm{C}), 134.4(\mathrm{C}), 130.0(\mathrm{CH}), 128.9(\mathrm{CH}), 128.7$ $(\mathrm{CH}), 128.3(\mathrm{CH}), 127.9(\mathrm{CH}), 127.6(\mathrm{CH}), 118.4(\mathrm{CN}), 89.4(\mathrm{C}), 64.8(\mathrm{CH}), 41.2\left(\mathrm{CH}_{2}\right)$ ppm; IR (neat): $\operatorname{vmax}\left(\mathrm{cm}^{-1}\right)=3028(\mathrm{~m}), 1489(\mathrm{~s}), 1450(\mathrm{~s}), 1339(\mathrm{~m}), 1157(\mathrm{~s}), 1068(\mathrm{~s}), 997(\mathrm{~s}), 903(\mathrm{~m}), 754(\mathrm{~s}), 696$ (s), 635 (s); HRMS (ESI): $m / z$ calculated for $\mathrm{C}_{28} \mathrm{H}_{23} \mathrm{NNaO}$ [M+Na] $]^{+} 412.1672$, found: 412.1658 .

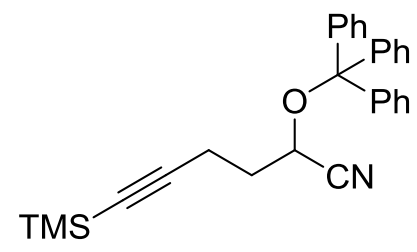

\section{6-(trimethylsilyl)-2-(trityloxy)hex-5-ynenitrile 3e}

Prepared from 5-(trimethylsilyl)pent-4-ynal $(93 \mathrm{mg}, 0.5 \mathrm{mmol}, 1$ equiv) and trityl isocyanide (148 $\mathrm{mg}$, $0.55 \mathrm{mmol}, 1.1$ equiv) according to Procedure $\mathrm{A}(5.5 \mathrm{~h}$ reaction time). Purification: column chromatography on silicagel (cyclohexane/EtOAc 100/1, $\mathrm{R}_{\mathrm{f}}=0.45$ in cyclohexane/EtOAc 10:1). Isolated as a white solid. Yield: $146 \mathrm{mg}, 0.345 \mathrm{mmol}, 69 \%$.

m.p.: 100-106 ${ }^{\circ} \mathrm{C} ;{ }^{1} \mathrm{H}-\mathrm{NMR}\left(\mathrm{CDCl}_{3}, 500 \mathrm{MHz}\right): \delta$ 7.52-7.48 (m, 6H), $7.36(\mathrm{tt}, \mathrm{J}=7.6,1.6 \mathrm{~Hz}, 6 \mathrm{H}), 7.32$ $(\mathrm{tt}, \mathrm{J}=6.9,2.2 \mathrm{~Hz}, 3 \mathrm{H}), 4.29(\mathrm{dd}, \mathrm{J}=6.9,5.4 \mathrm{~Hz}, 1 \mathrm{H}), 2.54(\mathrm{ddd}, \mathrm{J}=17.3,7.9,6.0 \mathrm{~Hz}, 1 \mathrm{H}), 2.43(\mathrm{dt}, \mathrm{J}=$ 17.3, $7.9 \mathrm{~Hz}, 1 \mathrm{H}), 2.12-2.00(\mathrm{~m}, 2 \mathrm{H}), 0.10(\mathrm{~s}, 9 \mathrm{H}) \mathrm{ppm} ;{ }^{13} \mathrm{C}\left\{{ }^{1} \mathrm{H}\right\}-\mathrm{NMR}\left(\mathrm{CDCl}_{3}, 125 \mathrm{MHz}\right): \delta 142.7(\mathrm{C})$, $128.8(\mathrm{CH}), 128.3(\mathrm{CH}), 128.0(\mathrm{CH}), 118.1(\mathrm{CN}), 104.4(\mathrm{C}), 89.2(\mathrm{C}), 86.3(\mathrm{C}), 62.2(\mathrm{CH}), 33.7\left(\mathrm{CH}_{2}\right)$, $15.7\left(\mathrm{CH}_{2}\right), 0.1\left(\mathrm{CH}_{3}\right)$ ppm; IR (neat): vmax $\left(\mathrm{cm}^{-1}\right)=3059(\mathrm{~m}), 2996(\mathrm{~m}), 2177$ (s), 1489 (s), 1447 (s), 1250 (s), 1084 (s), 905 (s), 837 (m), 750 (s), 706 (s); HRMS (ESI): m/z calculated for $\mathrm{C}_{28} \mathrm{H}_{29} \mathrm{NNaOSi}$ $[\mathrm{M}+\mathrm{Na}]^{+}$446.1911, found: 446.1912 . 
<smiles>N#CC(OC(c1ccccc1)(c1ccccc1)c1ccccc1)C1CC1</smiles>

\section{2-cyclopropyl-2-(trityloxy)acetonitrile $3 f$}

Prepared from cyclopropanecarboxaldehyde (35 mg, $0.5 \mathrm{mmol}, 1$ equiv) and trityl isocyanide (148 $\mathrm{mg}$, $0.55 \mathrm{mmol}, 1.1$ equiv) according to Procedure $\mathrm{A}(6 \mathrm{~h}$ reaction time). Purification: column chromatography on silicagel (cyclohexane/EtOAc 100/1, $\mathrm{R}_{\mathrm{f}}=0.39$ in cyclohexane/EtOAc 10:1). Isolated as a white solid. Yield: $85 \mathrm{mg}, 0.250 \mathrm{mmol}, 50 \%$.

m.p.: $106-111^{\circ} \mathrm{C} ;{ }^{1} \mathrm{H}-\mathrm{NMR}\left(\mathrm{CDCl}_{3}, 500 \mathrm{MHz}\right): \delta$ 7.58-7.53 (m, 6H), 7.40-7.35 (m, 6H), $7.32(\mathrm{tt}, \mathrm{J}=7.3$, $2.5 \mathrm{~Hz}, 3 \mathrm{H}), 3.96(\mathrm{~d}, \mathrm{~J}=6.9 \mathrm{~Hz}, 1 \mathrm{H}), 1.40-1.32(\mathrm{~m}, 1 \mathrm{H}), 0.75-0.65(\mathrm{~m}, 2 \mathrm{H}), 0.57-0.51(\mathrm{~m}, 1 \mathrm{H}), 0.43-0.37$ $(\mathrm{m}, 1 \mathrm{H}) \mathrm{ppm} ;{ }^{13} \mathrm{C}\left\{{ }^{1} \mathrm{H}\right\}-\mathrm{NMR}\left(\mathrm{CDCl}_{3}, 125 \mathrm{MHz}\right): \delta 142.9(\mathrm{C}), 128.9(\mathrm{CH}), 128.2(\mathrm{CH}), 127.9(\mathrm{CH}), 117.3$ $(\mathrm{CN}), 88.8(\mathrm{C}), 66.7(\mathrm{CH}), 14.9(\mathrm{CH}), 4.6\left(\mathrm{CH}_{2}\right), 2.5\left(\mathrm{CH}_{2}\right) \mathrm{ppm}$; IR (neat): vmax $\left(\mathrm{cm}^{-1}\right)=3057(\mathrm{~m}), 3026$ (m), 1489 (s), 1448 (s), 1339 (m), 1157 (m), 1067 (s), 999 (s), 756 (s), 696 (s), 635 (s); HRMS (ESI): m/z calculated for $\mathrm{C}_{24} \mathrm{H}_{21} \mathrm{NNaO}[\mathrm{M}+\mathrm{Na}]^{+} 362.1515$, found: 362.1516 .<smiles>N#CC(OC(c1ccccc1)(c1ccccc1)c1ccccc1)C1CCCCC1</smiles>

\section{2-cyclohexyl-2-(trityloxy)acetonitrile 3g}

Prepared from cyclohexanecarbaldehyde (56 mg, $0.5 \mathrm{mmol}, 1$ equiv) and trityl isocyanide (148 $\mathrm{mg}, 0.55$ mmol, 1.1 equiv) according to Procedure A ( $5 \mathrm{~h}$ reaction time). Purification: column chromatography on silicagel (cyclohexane/EtOAc 100/1, $\mathrm{R}_{\mathrm{f}}=0.33$ in cyclohexane/EtOAc 10:1). Isolated as a white solid. Yield: $97 \mathrm{mg}, 0.255 \mathrm{mmol}, 51 \%$ (co-elutes with triphenylacetonitrile, reported yield is corrected for 85 wt $\%$ purity).

m.p.: 103-130 ${ }^{\circ} \mathrm{C} ;{ }^{1} \mathrm{H}-\mathrm{NMR}\left(\mathrm{CDCl}_{3}, 500 \mathrm{MHz}\right): \delta$ 7.60-7.53 (m, 6H), 7.42-7.36 (m, 6H), $7.34(\mathrm{tt}, \mathrm{J}=7.3$, $2.5 \mathrm{~Hz}, 3 \mathrm{H}), 3.97$ (d, J = 4.4 Hz, 1H), 2.16-2.09 (m, 1H), 1.90-1.83 (m, 1H), 1.83-1.67 (m, 2H), 1.64-1.54 (m, 2H), 1.39-1.12 (m, 5H) ppm; ${ }^{13} \mathrm{C}\left\{{ }^{1} \mathrm{H}\right\}-\mathrm{NMR}\left(\mathrm{CDCl}_{3}, 125 \mathrm{MHz}\right): \delta 143.1(\mathrm{C}), 128.8(\mathrm{CH}), 128.2$ $(\mathrm{CH}), 127.8(\mathrm{CH}), 117.8(\mathrm{CN}), 88.7(\mathrm{C}), 68.1(\mathrm{CH}), 42.0(\mathrm{CH}), 28.5\left(\mathrm{CH}_{2}\right), 27.6\left(\mathrm{CH}_{2}\right), 26.1\left(\mathrm{CH}_{2}\right), 25.8$ $\left(\mathrm{CH}_{2}\right), 25.7\left(\mathrm{CH}_{2}\right)$, ppm; IR (neat): vmax $\left(\mathrm{cm}^{-1}\right)=2926(\mathrm{~s}), 2854(\mathrm{~s}), 1489(\mathrm{~s}), 1447$ (s), $1228(\mathrm{~m}), 1045$ (s), 980 (s), 899 (s), 766 (s), 756 (s), 694 (s); HRMS (ESI): $\mathrm{m} / z$ calculated for $\mathrm{C}_{27} \mathrm{H}_{27} \mathrm{NNaO}[\mathrm{M}+\mathrm{Na}]^{+}$ 404.1985, found: 404.1976 . 
<smiles>CCCOC(=O)N1CCC(C(C#N)OC(c2ccccc2)(c2ccccc2)c2ccccc2)CC1</smiles>

\section{tert-butyl 4-(cyano(trityloxy)methyl)piperidine-1-carboxylate 3h}

Prepared from tert-butyl 4-formylpiperidine-1-carboxylate (110 mg, $0.51 \mathrm{mmol}, 1$ equiv) and trityl isocyanide (148 mg, $0.55 \mathrm{mmol}, 1.1$ equiv) according to Procedure A ( $24 \mathrm{~h}$ reaction time). Purification: column chromatography on silicagel (cyclohexane/EtOAc 100/1, $\mathrm{R}_{\mathrm{f}}=0.20$ in cyclohexane/EtOAc 10:1). Isolated as a colorless oil. Yield: $57 \mathrm{mg}, 0.117 \mathrm{mmol}, 23 \%$.

${ }^{1} \mathrm{H}-\mathrm{NMR}\left(\mathrm{CDCl}_{3}, 500 \mathrm{MHz}\right): \delta$ 7.53-7.47 (m, 6H), 7.39-7.27 (m, 9H), $4.40(\mathrm{br}, 2 \mathrm{H}), 3.95(\mathrm{~d}, \mathrm{~J}=4.7 \mathrm{~Hz}$, $1 \mathrm{H}), 2.60$ (br, 2H), 1.98-1.87 (m, 1H), 1.72-1.58 (m, 1H), 1.57-1.39 (m, 2H), $1.46(\mathrm{~s}, 9 \mathrm{H}), 1.39-1.22(\mathrm{~m}$, 1H). ppm; ${ }^{13} \mathrm{C}\left\{{ }^{1} \mathrm{H}\right\}$-NMR $\left(\mathrm{CDCl}_{3}, 125 \mathrm{MHz}\right): \delta 154.7(\mathrm{CO}), 142.8(\mathrm{C}), 128.8(\mathrm{CH}), 128.3(\mathrm{CH}), 128.0$ $(\mathrm{CH}), 117.3(\mathrm{CN}), 89.0(\mathrm{C}), 79.7(\mathrm{C}), 67.1(\mathrm{CH}), 40.6(\mathrm{CH}), 39.0\left(\mathrm{CH}_{2}\right), 29.8\left(\mathrm{CH}_{2}\right), 28.5\left(\mathrm{CH}_{3}\right), 27.5$ $\left(\mathrm{CH}_{2}\right), 27.0\left(\mathrm{CH}_{2}\right)$ ppm; IR (neat): vmax $\left(\mathrm{cm}^{-1}\right)=2930(\mathrm{~m}), 1684(\mathrm{~s}), 1489$ (s), 1449 (s), 1053 (s), $978(\mathrm{~s})$, 901 (s), 767 (s), 698 (s), 644 (s), 631 (s); HRMS (ESI): $\mathrm{m} / z$ calculated for $\mathrm{C}_{31} \mathrm{H}_{34} \mathrm{~N}_{2} \mathrm{NaO}_{3}[\mathrm{M}+\mathrm{Na}]^{+}$ 505.2462, found: 505.2452 .

Note: most likely conformational changes (ring flip, N-Boc rotation) occur on the NMR time scale which determine the broadening of some signals in ${ }^{1} \mathrm{H}-\mathrm{NMR}$ and lower the intensity of some signals in ${ }^{13} \mathrm{C}$ NMR.<smiles>CC(C)C(C#N)OC(c1ccccc1)(c1ccccc1)c1ccccc1</smiles>

\section{3-methyl-2-(trityloxy)butanenitrile 3i}

Prepared from isobutyraldehyde (36 mg, $0.5 \mathrm{mmol}, 1$ equiv) and trityl isocyanide (148 $\mathrm{mg}, 0.55 \mathrm{mmol}$, 1.1 equiv) according to Procedure $\mathrm{A}$ ( $5 \mathrm{~h}$ reaction time). Purification: column chromatography on silicagel (cyclohexane/EtOAc 100/1, $\mathrm{R}_{\mathrm{f}}=0.38$ in cyclohexane/EtOAc 10:1). Isolated as a white solid. Yield: $123 \mathrm{mg}, 0.360 \mathrm{mmol}, 72 \%$.

m.p.: $105-109^{\circ} \mathrm{C} ;{ }^{1} \mathrm{H}-\mathrm{NMR}\left(\mathrm{CDCl}_{3}, 500 \mathrm{MHz}\right): \delta 7.58-7.52(\mathrm{~m}, 6 \mathrm{H}), 7.42-7.36(\mathrm{~m}, 6 \mathrm{H}), 7.33$ $(\mathrm{tt}, \mathrm{J}=7.3,2.5 \mathrm{~Hz}, 3 \mathrm{H}), 4.02(\mathrm{~d}, \mathrm{~J}=4.4 \mathrm{~Hz}, 1 \mathrm{H}), 2.01-1.90(\mathrm{~m}, 1 \mathrm{H}), 1.22(\mathrm{~d}, \mathrm{~J}=6.6 \mathrm{~Hz}, 3 \mathrm{H}), 0.97(\mathrm{~d}, \mathrm{~J}=$ $6.9 \mathrm{~Hz}, 3 \mathrm{H}) \mathrm{ppm} ;{ }^{13} \mathrm{C}\left\{{ }^{1} \mathrm{H}\right\}-\mathrm{NMR}\left(\mathrm{CDCl}_{3}, 125 \mathrm{MHz}\right): \delta 143.0(\mathrm{C}), 128.8(\mathrm{CH}), 128.2(\mathrm{CH}), 127.8(\mathrm{CH})$, $117.3(\mathrm{CN}), 88.8(\mathrm{C}), 68.8(\mathrm{CH}), 32.7(\mathrm{CH}), 18.2\left(\mathrm{CH}_{3}\right), 17.1\left(\mathrm{CH}_{3}\right) \mathrm{ppm}$; IR (neat): vmax $\left(\mathrm{cm}^{-1}\right)=2934$ (m), 1489 (s), 1447 (s), 1354 (s), 1213 (s), 1053 (s), 1030 (s), 980 (s), 901 (s), 766 (s), 631 (s); HRMS (ESI): $m / z$ calculated for $\mathrm{C}_{24} \mathrm{H}_{23} \mathrm{NNaO}[\mathrm{M}+\mathrm{Na}]^{+} 364.1672$, found: 364.1667 . 
<smiles>CC(c1ccccc1)C(C#N)OC(c1ccccc1)(c1ccccc1)c1ccccc1</smiles>

\section{3-phenyl-2-(trityloxy)butanenitrile 3j}

Prepared from 2-phenylpropanal (67 mg, $0.5 \mathrm{mmol}, 1$ equiv) and trityl isocyanide (148 $\mathrm{mg}, 0.55 \mathrm{mmol}$, 1.1 equiv) according to Procedure $\mathrm{A}(5 \mathrm{~h}$ reaction time). Purification: column chromatography on silicagel (cyclohexane/EtOAc 100/1, $\mathrm{R}_{\mathrm{f}}=0.30$ in cyclohexane/EtOAc 10:1). Diastereoisomers (formed in $\sim 1: 1$ ratio) were only partly resolved. Isolated as white amorphous solids. Combined yield: $122 \mathrm{mg}, 0.345$ mmol, $69 \%$ (co-elutes with triphenylacetonitrile, reported yield is corrected for $90 \mathrm{wt} \%$ purity).

Fraction 1 (20:1 ratio, relative configuration not assigned): ${ }^{1} \mathrm{H}-\mathrm{NMR}\left(\mathrm{CDCl}_{3}, 500 \mathrm{MHz}\right): \delta$ 7.53-7.49 (m, 6H), 7.44-7.17 (m, 12H), 7.10-7.05 (m, 2H), 4.24 (dd, J = 5.0 Hz, 1H), 2.86-2.77 (m, 1H), 1.56 (d, J = 6.9 $\mathrm{Hz}, 3 \mathrm{H}) \mathrm{ppm} ;{ }^{13} \mathrm{C}\left\{{ }^{1} \mathrm{H}\right\}-\mathrm{NMR}\left(\mathrm{CDCl}_{3}, 125 \mathrm{MHz}\right): \delta 143.0(\mathrm{C}), 139.6(\mathrm{C}), 128.9(\mathrm{CH}), 128.7(\mathrm{CH}), 128.3$ $(\mathrm{CH}), 128.1(\mathrm{CH}), 127.9(\mathrm{CH}), 127.6(\mathrm{CH}), 117.4(\mathrm{CN}), 89.5(\mathrm{C}), 69.6(\mathrm{CH}), 43.3(\mathrm{CH}), 14.5\left(\mathrm{CH}_{3}\right) \mathrm{ppm}$;

Fraction 2 (1:5 ratio, relative configuration not assigned): ${ }^{1} \mathrm{H}-\mathrm{NMR}\left(\mathrm{CDCl}_{3}, 500 \mathrm{MHz}\right): \delta$ 7.44-7.27 (m, $20 \mathrm{H}), 4.21(\mathrm{dd}, \mathrm{J}=5.0 \mathrm{~Hz}, 1 \mathrm{H}), 3.07-3.00(\mathrm{~m}, 1 \mathrm{H}), 1.40(\mathrm{~d}, \mathrm{~J}=7.3 \mathrm{~Hz}, 3 \mathrm{H}) \mathrm{ppm} ;{ }^{13} \mathrm{C}\left\{{ }^{1} \mathrm{H}\right\}-\mathrm{NMR}\left(\mathrm{CDCl}_{3}\right.$, $125 \mathrm{MHz}): \delta 142.8(\mathrm{C}), 139.9(\mathrm{C}), 128.9(\mathrm{CH}), 128.7(\mathrm{CH}), 128.4(\mathrm{CH}), 128.2(\mathrm{CH}), 127.8(\mathrm{CH}), 127.6$ $(\mathrm{CH}), 117.6(\mathrm{CN}), 89.0(\mathrm{C}), 68.2(\mathrm{CH}), 43.3(\mathrm{CH}), 16.1\left(\mathrm{CH}_{3}\right) \mathrm{ppm}$;

IR (neat): vmax $\left(\mathrm{cm}^{-1}\right)=2926$ (s), 2854 (s), 1489 (s), 1448 (s), 1340 (m), 1223 (s), 1045 (s), 978 (s), 899 (s), 766 (s), 694 (s); HRMS (ESI): $m / z$ calculated for $\mathrm{C}_{29} \mathrm{H}_{25} \mathrm{NNaO}[\mathrm{M}+\mathrm{Na}]^{+} 426.1828$, found: 426.1823.<smiles>CC(C)(C)C(C#N)OC(c1ccccc1)(c1ccccc1)c1ccccc1</smiles>

\section{3,3-dimethyl-2-(trityloxy)butanenitrile 3k}

Prepared from pivaldehyde ( $43 \mathrm{mg}, 0.5 \mathrm{mmol}, 1$ equiv) and trityl isocyanide (148 $\mathrm{mg}, 0.55 \mathrm{mmol}, 1.1$ equiv) according to Procedure A ( $5 \mathrm{~h}$ reaction time). Purification: column chromatography on silicagel (cyclohexane/EtOAc 100/1, $\mathrm{R}_{\mathrm{f}}=0.47$ in cyclohexane/EtOAc 10:1). Isolated as a white solid. Yield: 96 $\mathrm{mg}, 0.270 \mathrm{mmol}, 54 \%$ (co-elutes with triphenylacetonitrile, reported yield is corrected for $90 \mathrm{wt} \%$ purity).

m.p.: 90-105 ${ }^{\circ} \mathrm{C} ;{ }^{1} \mathrm{H}-\mathrm{NMR}\left(\mathrm{CDCl}_{3}, 500 \mathrm{MHz}\right): \delta$ 7.55-7.50 (m, 6H), 7.35-7.30 (m, 6H), 7.27 (tt, J = 7.6 $\mathrm{Hz}, 1.6 \mathrm{~Hz}, 3 \mathrm{H}), 3.29$ (s, 1H), 1.07 (s, 9H) ppm; ${ }^{13} \mathrm{C}\left\{{ }^{1} \mathrm{H}\right\}-\mathrm{NMR}\left(\mathrm{CDCl}_{3}, 125 \mathrm{MHz}\right): \delta 143.1(\mathrm{C}), 129.0$ $(\mathrm{CH}), 128.1(\mathrm{CH}), 127.8(\mathrm{CH}), 118.1(\mathrm{CN}), 88.5(\mathrm{C}), 71.4(\mathrm{CH}), 36.3(\mathrm{C}), 26.0\left(\mathrm{CH}_{3}\right)$ ppm; IR (neat): $\operatorname{vmax}\left(\mathrm{cm}^{-1}\right)=2968(\mathrm{~m}), 1595(\mathrm{~m}), 1489(\mathrm{~s}), 1445(\mathrm{~s}), 1182(\mathrm{~m}), 1063(\mathrm{~s}), 1030(\mathrm{~s}), 901$ (s), $748(\mathrm{~s}), 696$ (s), 642 (s); HRMS (ESI): $m / z$ calculated for $\mathrm{C}_{25} \mathrm{H}_{25} \mathrm{NNaO}$ [M+Na] ${ }^{+} 378.1828$, found: 378.1819. 
<smiles>N#CC(OC(c1ccccc1)(c1ccccc1)c1ccccc1)c1ccccc1</smiles>

\section{2-phenyl-2-(trityloxy)acetonitrile 3m}

Prepared from benzaldehyde $(53 \mathrm{mg}, 0.5 \mathrm{mmol}, 1$ equiv) and trityl isocyanide (148 $\mathrm{mg}, 0.55 \mathrm{mmol}, 1.1$ equiv) according to Procedure A ( $7 \mathrm{~h}$ reaction time). Purification: column chromatography on silicagel (cyclohexane/EtOAc 100/1, $\mathrm{R}_{\mathrm{f}}=0.36$ in cyclohexane/EtOAc 10:1). Isolated as a white solid. Yield: 49 $\mathrm{mg}, 0.130 \mathrm{mmol}, 26 \%$.

m.p.: 116-118 ${ }^{\circ} \mathrm{C} ;{ }^{1} \mathrm{H}-\mathrm{NMR}\left(\mathrm{CDCl}_{3}, 500 \mathrm{MHz}\right): \delta$ 7.58-7.51 (m, 6H), 7.48-7.29 (m, 14H), $5.16(\mathrm{~s}, 1 \mathrm{H})$ ppm; ${ }^{13} \mathrm{C}\left\{{ }^{1} \mathrm{H}\right\}$-NMR $\left(\mathrm{CDCl}_{3}, 125 \mathrm{MHz}\right): \delta 142.7(\mathrm{C}), 135.6(\mathrm{C}), 129.4(\mathrm{CH}), 129.1(\mathrm{CH}), 128.9(\mathrm{CH})$, $128.3(\mathrm{CH}), 128.0(\mathrm{CH}), 127.1(\mathrm{CH}), 118.0(\mathrm{CN}), 90.0(\mathrm{C}), 65.2(\mathrm{CH}) \mathrm{ppm}$; IR (neat): vmax $\left(\mathrm{cm}^{-1}\right)=$ 3059 (m), 2177 (m), 1489 (s), 1448 (s), 1217 (m), 1082 (s), 1051 (s), 972 (s), 905 (s), 839 (s), 694 (s); HRMS (ESI): $m / z$ calculated for $\mathrm{C}_{27} \mathrm{H}_{21} \mathrm{NNaO}[\mathrm{M}+\mathrm{Na}]^{+} 398.1515$, found: 398.1499 .

Enantioselective reaction: prepared from benzaldehyde ( $27 \mathrm{mg}, 0.25 \mathrm{mmol}, 1$ equiv) and trityl isocyanide (135 mg, $0.5 \mathrm{mmol}, 2$ equiv) according to Procedure E. Purification: column chromatography on silicagel (cyclohexane/EtOAc 30/1, $\mathrm{R}_{\mathrm{f}}=0.2$ in cyclohexane/EtOAc 30:1). Isolated as a white solid. Yield: $47 \mathrm{mg}$, $0.125 \mathrm{mmol}, 50 \%$.

Chiral HPLC (Chiracel OD-H, heptane/isopropanol $=95 / 5, \mathrm{v}=0.5 \mathrm{~mL} / \mathrm{min}$, column temperature: $20{ }^{\circ} \mathrm{C}$, $\lambda=214 \mathrm{~nm}) \mathrm{t}($ major $)-3 \mathrm{~m}=14.0, \mathrm{t}($ minor $)-3 \mathrm{~m}=15.2 \mathrm{~min}, 79 \%$ ee.<smiles>CCCCCCC(O)C#N</smiles>

\section{2-hydroxyoctanenitrile 4b}

Prepared from heptaldehyde (57 mg, $0.5 \mathrm{mmol}, 1$ equiv) and trityl isocyanide (148 $\mathrm{mg}, 0.55 \mathrm{mmol}, 1.1$ equiv) according to Procedure B (5 h reaction time). Purification: column chromatography on silicagel (cyclohexane/EtOAc 10/1, $\mathrm{R}_{\mathrm{f}}=0.16$ ). Isolated as a pale yellow oil. Yield: $49 \mathrm{mg}, 0.350 \mathrm{mmol}, 70 \%$. Spectra in accordance to literature report. ${ }^{3}$

${ }^{1} \mathrm{H}-\mathrm{NMR}\left(\mathrm{CDCl}_{3}, 500 \mathrm{MHz}\right): \delta 4.48(\mathrm{t}, \mathrm{J}=7.0 \mathrm{~Hz}, 1 \mathrm{H}), 3.43(\mathrm{br}, 1 \mathrm{H}), 1.86-1.82(\mathrm{~m}, 2 \mathrm{H}), 1.54-1.43(\mathrm{~m}$, $2 \mathrm{H}), 1.39-1.24(\mathrm{~m}, 6 \mathrm{H}), 0.88(\mathrm{t}, \mathrm{J}=6.8 \mathrm{~Hz}, 3 \mathrm{H}) \mathrm{ppm} ;{ }^{13} \mathrm{C}\left\{{ }^{1} \mathrm{H}\right\}-\mathrm{NMR}\left(\mathrm{CDCl}_{3}, 125 \mathrm{MHz}\right): \delta 119.9(\mathrm{CN})$, $61.4(\mathrm{CH}), 35.3\left(\mathrm{CH}_{2}\right), 31.6\left(\mathrm{CH}_{2}\right), 28.6\left(\mathrm{CH}_{2}\right), 24.5\left(\mathrm{CH}_{2}\right), 22.5\left(\mathrm{CH}_{2}\right), 14.2\left(\mathrm{CH}_{3}\right) \mathrm{ppm}$.<smiles>N#CC(O)C1CCCCC1</smiles>

\section{2-cyclohexyl-2-hydroxyacetonitrile $4 \mathrm{~g}$}


Prepared from cyclohexanecarbaldehyde ( $56 \mathrm{mg}, 0.5 \mathrm{mmol}, 1$ equiv) and trityl isocyanide (148 $\mathrm{mg}, 0.55$ mmol, 1.1 equiv) according to Procedure $\mathrm{B}$ ( $5 \mathrm{~h}$ reaction time). Purification: column chromatography on silicagel (cyclohexane/EtOAc 10/1, $\mathrm{R}_{\mathrm{f}}=0.37$ ). Isolated as a colorless oil. Yield: $63 \mathrm{mg}, 0.450 \mathrm{mmol}, 90 \%$ (co-elutes with triethylsilanol, reported yield is corrected for $97 \mathrm{wt} \%$ purity). Spectra in accordance to literature report. ${ }^{3}$

${ }^{1} \mathrm{H}-\mathrm{NMR}\left(\mathrm{CDCl}_{3}, 500 \mathrm{MHz}\right): \delta 4.28(\mathrm{t}, \mathrm{J}=6.0 \mathrm{~Hz}, 1 \mathrm{H}), 3.55(\mathrm{br}, 1 \mathrm{H}), 1.92-1.70(\mathrm{~m}, 6 \mathrm{H}), 1.33-1.03(\mathrm{~m}$, $5 \mathrm{H}) \mathrm{ppm} ;{ }^{13} \mathrm{C}\left\{{ }^{1} \mathrm{H}\right\}-\mathrm{NMR}\left(\mathrm{CDCl}_{3}, 125 \mathrm{MHz}\right): \delta 119.0(\mathrm{CN}), 66.5(\mathrm{CH}), 42.4(\mathrm{CH}), 28.1\left(\mathrm{CH}_{2}\right), 27.8$ $\left(\mathrm{CH}_{2}\right), 26.0\left(\mathrm{CH}_{2}\right), 25.5\left(\mathrm{CH}_{2}\right), 25.5\left(\mathrm{CH}_{2}\right) \mathrm{ppm}$.<smiles>N#CC(O)c1ccccc1</smiles>

\section{2-phenyl-2-hydroxyacetonitrile $4 \mathrm{~m}$}

Prepared from benzaldehyde (53 mg, $0.5 \mathrm{mmol}, 1$ equiv) and trityl isocyanide (148 $\mathrm{mg}, 0.55 \mathrm{mmol}, 1.1$ equiv) according to Procedure $\mathrm{B}$ ( $5 \mathrm{~h}$ reaction time). Purification: column chromatography on silicagel (cyclohexane/EtOAc 7/1, $\mathrm{R}_{\mathrm{f}}=0.13$ ). Isolated as a colorless oil. Yield: $20 \mathrm{mg}, 0.15 \mathrm{mmol}, 30 \%$. Spectra in accordance to literature report. ${ }^{3}$

${ }^{1} \mathrm{H}-\mathrm{NMR}\left(\mathrm{CDCl}_{3}, 500 \mathrm{MHz}\right): \delta$ 7.55-7.51 (m, 2H), 7.48-7.43 (m, 3H), $5.52(\mathrm{~s}, 1 \mathrm{H}), 3.50$ (br, 1H) ppm; ${ }^{13} \mathrm{C}\left\{{ }^{1} \mathrm{H}\right\}$-NMR $\left(\mathrm{CDCl}_{3}, 125 \mathrm{MHz}\right): \delta 135.3(\mathrm{C}), 129.9(\mathrm{CH}), 128.3(\mathrm{CH}), 126.7(\mathrm{CH}), 119.0(\mathrm{CN}), 63.6$ (CH) ppm.<smiles>CC1CC(=O)OC(C(=O)NC(c2ccccc2)(c2ccccc2)c2ccccc2)C1</smiles>

\section{4-methyl-1-oxo-1-(tritylamino)pentan-2-yl acetate 6a}

Prepared from isovaleraldehyde ( $43 \mathrm{mg}, 0.5 \mathrm{mmol}, 1$ equiv), acetic acid (30 mg, $0.5 \mathrm{mmol}, 1$ equiv) and trityl isocyanide (148 mg, $0.55 \mathrm{mmol}, 1.1$ equiv) according to Procedure C ( $24 \mathrm{~h}$ reaction time). Purification: column chromatography on silicagel (cyclohexane/EtOAc 10/1). Isolated as a white amorphous solid. Yield: $172 \mathrm{mg}, 0.415 \mathrm{mmol}, 83 \%$.

${ }^{1} \mathrm{H}-\mathrm{NMR}\left(\mathrm{CDCl}_{3}, 500 \mathrm{MHz}\right): \delta$ 7.37-7.31 (m, 6H), $7.29(\mathrm{tt}, \mathrm{J}=7.3,2.2 \mathrm{~Hz}, 3 \mathrm{H})$, 7.27-7.21 (m, 7H). 5.27 (dd, J = 9.1, 3.8 Hz, 1H), 2.19 (s, 3H), 1.82-1.66 (m, 3H), 0.97 (d, J = 6.3 Hz, 3H), 0.95 (d, J = 6.3 Hz, $3 \mathrm{H}) \mathrm{ppm} ;{ }^{13} \mathrm{C}\left\{{ }^{1} \mathrm{H}\right\}$-NMR $\left(\mathrm{CDCl}_{3}, 125 \mathrm{MHz}\right): \delta 170.0(\mathrm{CO}), 168.8(\mathrm{CO}), 144.4(\mathrm{C}), 128.5(\mathrm{CH}), 128.0$ $(\mathrm{CH}), 127.2(\mathrm{CH}), 73.4(\mathrm{CH}), 70.2(\mathrm{C}), 40.3\left(\mathrm{CH}_{2}\right), 24.5(\mathrm{CH}), 23.1\left(\mathrm{CH}_{3}\right), 21.7\left(\mathrm{CH}_{3}\right), 20.9\left(\mathrm{CH}_{3}\right) \mathrm{ppm}$; IR (neat): vmax $\left(\mathrm{cm}^{-1}\right)=2957(\mathrm{~m}), 1740(\mathrm{~s}), 1697(\mathrm{~s}), 1489(\mathrm{~m}), 1447(\mathrm{w}), 1369(\mathrm{~s}), 1215$ (s), $1068(\mathrm{~s})$, 1024 (m), 750 (m), 696 (s); HRMS (ESI): $\mathrm{m} / z$ calculated for $\mathrm{C}_{27} \mathrm{H}_{29} \mathrm{NNaO}_{3}[\mathrm{M}+\mathrm{Na}]^{+} 438.2040$, found: 438.2031 . 
<smiles>O=C(OC(C(=O)NC(c1ccccc1)(c1ccccc1)c1ccccc1)c1cccc([N+](=O)[O-])c1)c1ccccc1</smiles>

\section{1-(3-nitrophenyl)-2-oxo-2-(tritylamino)ethyl benzoate $6 \mathrm{~b}$}

Prepared from 3-nitrobenzaldehyde ( $76 \mathrm{mg}, 0.5 \mathrm{mmol}, 1$ equiv), benzoic acid (61 mg, $0.5 \mathrm{mmol}, 1$ equiv) and trityl isocyanide ( $148 \mathrm{mg}, 0.55 \mathrm{mmol}, 1.1$ equiv) according to Procedure $\mathrm{C}$ ( $24 \mathrm{~h}$ reaction time). Purification: column chromatography on silicagel (cyclohexane/EtOAc $10 / 1$ to $3: 1, \mathrm{R}_{\mathrm{f}}=0.50$ in cyclohexane/EtOAc 3:1). Isolated as a white solid. Yield: $201 \mathrm{mg}, 0.370 \mathrm{mmol}, 74 \%$.

m.p.: 82-83.7 ${ }^{\circ} \mathrm{C} ;{ }^{1} \mathrm{H}-\mathrm{NMR}\left(\mathrm{CDCl}_{3}, 500 \mathrm{MHz}\right): \delta 8.41(\mathrm{~s}, 1 \mathrm{H}), 8.25(\mathrm{dd}, \mathrm{J}=8.2,1.3 \mathrm{~Hz}, 1 \mathrm{H}), 8.21(\mathrm{~d}, \mathrm{~J}=$ $7.5 \mathrm{~Hz}, 2 \mathrm{H}), 7.93(\mathrm{~s}, 1 \mathrm{H}), 7.88(\mathrm{~d}, \mathrm{~J}=7.9 \mathrm{~Hz}, 1 \mathrm{H}), 7.72(\mathrm{t}, \mathrm{J}=7.3 \mathrm{~Hz}, 1 \mathrm{H}), 7.61-7.52(\mathrm{~m}, 3 \mathrm{H}), 7.39-7.25$ (m, 15H), $6.50(\mathrm{~s}, 1 \mathrm{H}) \mathrm{ppm} ;{ }^{13} \mathrm{C}\left\{{ }^{1} \mathrm{H}\right\}-\mathrm{NMR}\left(\mathrm{CDCl}_{3}, 125 \mathrm{MHz}\right): \delta 166.0(\mathrm{CO}), 164.6(\mathrm{CO}), 148.2(\mathrm{C})$, $144.0(\mathrm{C}), 137.2(\mathrm{C}), 134.2(\mathrm{CH}), 133.9(\mathrm{CH}), 129.8(\mathrm{CH}), 129.7(\mathrm{CH}), 128.8(\mathrm{CH}), 128.5(\mathrm{CH}), 128.1$ $(\mathrm{CH}), 127.3(\mathrm{CH}), 123.8(\mathrm{CH}), 122.2(\mathrm{CH}), 75.0(\mathrm{CH}), 70.7(\mathrm{C}) \mathrm{ppm}$; ; IR (neat): vmax $\left(\mathrm{cm}^{-1}\right)=1701(\mathrm{~s})$, 1680 (s), 1528 (s), 1489 (s), 1349 (s), 1244 (s), 1026 (m), 698 (s); HRMS (ESI): m/z calculated for $\mathrm{C}_{34} \mathrm{H}_{26} \mathrm{~N}_{2} \mathrm{NaO}_{5}[\mathrm{M}+\mathrm{Na}]^{+}$565.1734, found: 565.1708 .

Note: two quaternary carbon signals overlap.<smiles>CC(=O)OC(C(=O)NC(c1ccccc1)(c1ccccc1)c1ccccc1)c1ccc(Cl)cc1</smiles>

\section{1-(4-chlorophenyl)-2-oxo-2-(tritylamino)ethyl acetate $6 \mathrm{c}$}

Prepared from $p$-chlorobenzaldehyde ( $71 \mathrm{mg}, 0.5 \mathrm{mmol}, 1$ equiv), acetic acid (30 mg, $0.5 \mathrm{mmol}, 1$ equiv) and trityl isocyanide ( $148 \mathrm{mg}, 0.55 \mathrm{mmol}, 1.1$ equiv) according to Procedure $\mathrm{C}$ ( $23 \mathrm{~h}$ reaction time). Purification: column chromatography on silicagel (cyclohexane/EtOAc $10 / 1$ to $4: 1, \mathrm{R}_{\mathrm{f}}=0.32$ in cyclohexane/EtOAc 4:1). Isolated as a white solid. Yield: $139 \mathrm{mg}, 0.295 \mathrm{mmol}, 59 \%$.

m.p.: 57.3-75.5 ${ }^{\circ} \mathrm{C} ;{ }^{1} \mathrm{H}-\mathrm{NMR}\left(\mathrm{CDCl}_{3}, 400 \mathrm{MHz}\right): \delta$ 7.42-7.28 (m, 14H), $7.18(\mathrm{~d}, \mathrm{~J}=7.7 \mathrm{~Hz}, 6 \mathrm{H}), 6.05(\mathrm{~s}$, $1 \mathrm{H}), 2.24(\mathrm{~s}, 3 \mathrm{H}) \mathrm{ppm} ;{ }^{13} \mathrm{C}\left\{{ }^{1} \mathrm{H}\right\}-\mathrm{NMR}\left(\mathrm{CDCl}_{3}, 125 \mathrm{MHz}\right): \delta 169.4(\mathrm{CO}), 166.2(\mathrm{CO}), 144.2(\mathrm{C}), 135.1$ $(\mathrm{C}), 133.6(\mathrm{C}), 129.0(\mathrm{CH}), 128.9(\mathrm{CH}), 128.6(\mathrm{CH}), 128.2(\mathrm{CH}), 127.3(\mathrm{CH}), 75.4(\mathrm{CH}), 70.6(\mathrm{C}), 21.1$ $\left(\mathrm{CH}_{3}\right)$ ppm; IR (neat): vmax $\left(\mathrm{cm}^{-1}\right)=3315$ (s), 3044 (s), 2388 (s), 1755 (m), 1682 (w), 1496 (w), 1244 (w), $1166(\mathrm{~m}), 7.54(\mathrm{~m}), 698(\mathrm{w}), 623(\mathrm{~s})$; HRMS (ESI): $\mathrm{m} / z$ calculated for $\mathrm{C}_{29} \mathrm{H}_{24} \mathrm{ClNO}_{3}[\mathrm{M}+\mathrm{H}]^{+}$ 470.1517, found: 470.1497 . 
<smiles>CC1(C)OC2OC(C(=O)Nc3ccccc3)C(OC(=O)c3ccccc3)C(O2)C1n1ccc(=O)[nH]c1=O</smiles>

\section{Passerini product 6d}

Prepared from aldehyde 11 (142 mg, $0.5 \mathrm{mmol}, 1$ equiv), benzoic acid (62 mg, $0.5 \mathrm{mmol}, 1$ equiv) and trityl isocyanide (148 mg, $0.55 \mathrm{mmol}, 1.1$ equiv) according to Procedure $\mathrm{C}$ ( $48 \mathrm{~h}$ reaction time). Purification: column chromatography on silicagel (cyclohexane/EtOAc 1/1, $\mathrm{R}_{\mathrm{f}}=0.16$ ). Diastereoisomers (formed in 1.5:1 ratio) were not resolved. Isolated as a white solid. Combined yield: $215 \mathrm{mg}, 0.320 \mathrm{mmol}$, $64 \%$.

m.p.: $127-153{ }^{\circ} \mathrm{C} ;{ }^{1} \mathrm{H}-\mathrm{NMR}\left(\mathrm{CDCl}_{3}, 500 \mathrm{MHz}\right.$; D1:D2 ratio 1.5:1): $\delta 9.8$ (s, NH, D1), 9.7 (s, NH, D2), 8.10-8.03 (m, 2H, D1, 2H, D2), 7.70 (s, NH, D1), 7.67-7.58 (m, 1H, D1, 1H, D2), 7.56 (s, NH, D2), 7.52$7.44(\mathrm{~m}, 2 \mathrm{H}, D 1,2 \mathrm{H}, D 2), 7.35(\mathrm{~d}, \mathrm{~J}=7.9 \mathrm{~Hz}, 1 \mathrm{H}, D 1), 7.31-7.18(\mathrm{~m}, 15 \mathrm{H}, D 1,15 \mathrm{H}, D 2), 7.16(\mathrm{~d}, \mathrm{~J}=$ $8.20 \mathrm{~Hz}, 1 \mathrm{H}, D 2), 5.85-5.78(\mathrm{~m}, 2 \mathrm{H}, D 1,2 \mathrm{H}, D 2), 5.57(\mathrm{~d}, \mathrm{~J}=7.9 \mathrm{~Hz}, 1 \mathrm{H}, D 1), 5.52(\mathrm{~d}, \mathrm{~J}=7.9 \mathrm{~Hz}, 1 \mathrm{H}$, D2), $5.19(\mathrm{dd}, \mathrm{J}=6.3,3.5 \mathrm{~Hz}, 1 \mathrm{H}, \mathrm{D} 2), 5.01(\mathrm{dd}, \mathrm{J}=6.3,3.8 \mathrm{~Hz}, 1 \mathrm{H}, D 1), 4.96(\mathrm{dd}, \mathrm{J}=6.3,3.2 \mathrm{~Hz}, 1 \mathrm{H}$, D2), $4.88(\mathrm{dd}, \mathrm{J}=6.3,2.8 \mathrm{~Hz}, 1 \mathrm{H}, D 1), 4.73(\mathrm{t}, \mathrm{J}=3.8 \mathrm{~Hz}, 1 \mathrm{H}, D 1), 4.65$ (t, J = 3.8 Hz, 1H, D2), 1.61 (s, $3 \mathrm{H}, D 2), 1.60$ (s, 3H, D1), 1.37 (s, 3H, D2), 1.36 (s, 3H,Dl) ppm; ${ }^{13} \mathrm{C}\left\{{ }^{1} \mathrm{H}\right\}-\mathrm{NMR}\left(\mathrm{CDCl}_{3}, 125 \mathrm{MHz}\right): \delta$ 165.6 (CO, D1), 165.5 (CO, D2), 165.2 (CO, D1), 165.1 (CO, D2), 163.7 (CO, D1), 163.4 (CO, D2), 150.22 (C, D1 and D2),144.1 (C, D2), $144.0(\mathrm{C}, D 1), 141.9(\mathrm{CH}, D 1$ and D2), $134.2(\mathrm{CH}, D 1), 133.9$ $(\mathrm{CH}, D 2), 129.9(\mathrm{CH}, D 1$ and D2), $128.8(\mathrm{CH}, \mathrm{D} 1), 128.7(\mathrm{C}, \mathrm{D} 2), 128.7(\mathrm{CH}, \mathrm{D} 2) 128.6(\mathrm{CH}, \mathrm{D} 2)$, $128.5(\mathrm{CH}, D 1), 128.4(\mathrm{C}, \mathrm{Dl}), 128.1(\mathrm{CH}, \mathrm{Dl}), 128.0(\mathrm{CH}, \mathrm{D} 2), 127.3(\mathrm{CH}, \mathrm{D} 1), 127.2$ (CH, D2), 115.1 (C, D2), 114.8 (C, D1), $103.1(\mathrm{CH}$, D2), $102.7(\mathrm{CH}$, D1), 93.7 (CH, D2), $93.0(\mathrm{CH}$, D1), 85.3 (CH, D1), $85.0(\mathrm{CH}, \mathrm{D} 2), 84.1(\mathrm{CH}, \mathrm{D} 1), 83.3(\mathrm{CH}, \mathrm{D} 2), 81.3(\mathrm{CH}, \mathrm{D} 2), 80.3(\mathrm{CH}, \mathrm{D} 1), 73.4(\mathrm{CH}, \mathrm{D} 2), 73.3(\mathrm{CH}$, D1), 70.8 (C, D2), 70.7 (C, D1), $27.2\left(\mathrm{CH}_{3}, \mathrm{Dl}\right.$ and D2), $25.4\left(\mathrm{CH}_{3}, \mathrm{Dl}\right), 25.3\left(\mathrm{CH}_{3}, \mathrm{D} 2\right) \mathrm{ppm}$; IR (neat): $\operatorname{vmax}\left(\mathrm{cm}^{-1}\right)=3059(\mathrm{w}), 1678$ (br), 1491 (s), 1448 (s), 1379 (s), 1246 (br), 1084 (s), 1067 (s), 905 (m), 810 (m), 698 (s); HRMS (ESI): $m / z$ calculated for $\mathrm{C}_{39} \mathrm{H}_{35} \mathrm{~N}_{3} \mathrm{NaO}_{8}[\mathrm{M}+\mathrm{Na}]^{+}$696.2316, found: 696.2294 .<smiles>CC1CC(=O)OC(C(N)=O)C1</smiles>

\section{2-amino-1-(4-chlorophenyl)-2-oxoethyl acetate 7a}

Prepared from 6a (75 mg, $0.18 \mathrm{mmol}$ ) according to Procedure D (4 h reaction time). Purification: column chromatography on silicagel (cyclohexane/EtOAc 1/1, $\mathrm{R}_{\mathrm{f}}=0.24$ ). Isolated as a white solid. Yield: $19 \mathrm{mg}$, $0.108 \mathrm{mmol}, 60 \%$. 
m.p.: 69-73 ${ }^{\circ} \mathrm{C} ;{ }^{1} \mathrm{H}-\mathrm{NMR}\left(\mathrm{CDCl}_{3}, 500 \mathrm{MHz}\right): \delta 6.04$ (s, 2H), $5.18(\mathrm{dd}, \mathrm{J}=9.1,3.8 \mathrm{~Hz}, 1 \mathrm{H}), 2.15(\mathrm{~s}, 3 \mathrm{H})$, 1.80-1.65 (m, 3H), $0.94(\mathrm{~d}, \mathrm{~J}=6.0 \mathrm{~Hz}, 3 \mathrm{H}), 0.92(\mathrm{~d}, \mathrm{~J}=6.0 \mathrm{~Hz}, 3 \mathrm{H}) \mathrm{ppm} ;{ }^{13} \mathrm{C}\left\{{ }^{1} \mathrm{H}\right\}-\mathrm{NMR}\left(\mathrm{CDCl}_{3}, 125\right.$ MHz): $\delta 173.3(\mathrm{CO}), 170.1(\mathrm{CO}), 72.6(\mathrm{CH}), 40.8\left(\mathrm{CH}_{2}\right), 24.6(\mathrm{CH}), 23.2\left(\mathrm{CH}_{3}\right), 21.9\left(\mathrm{CH}_{3}\right), 21.1\left(\mathrm{CH}_{3}\right)$ ppm; IR (neat): vmax $\left(\mathrm{cm}^{-1}\right)=3032(\mathrm{w}), 2233(\mathrm{w}), 1684(\mathrm{~m}), 1593(\mathrm{~m}), 1489(\mathrm{~s}), 1445$ (s), 1082 (s), 1032 (s), 750 (s), 694 (s), 642 (s); HRMS (ESI): $\mathrm{m} / z$ calculated for $\mathrm{C}_{8} \mathrm{H}_{15} \mathrm{NNaO}_{3}[\mathrm{M}+\mathrm{Na}]^{+}$196.0944, found: 196.0941 .<smiles>NC(=O)C(OC(=O)c1ccccc1)c1cccc([N+](=O)[O-])c1</smiles>

\section{2-amino-1-(3-nitrophenyl)-2-oxoethyl benzoate 7b}

Prepared from 6b (109 mg, $0.20 \mathrm{mmol})$ according to Procedure D (20 h reaction time). Purification: trituration with pentane $\left(\mathrm{R}_{\mathrm{f}}=0.24\right.$ in cyclohexane/EtOAc 1:1). Isolated as a white solid. Yield: $120 \mathrm{mg}$, $0.400 \mathrm{mmol}, 80 \%$.

m.p.: 169-173 ${ }^{\circ} \mathrm{C} ;{ }^{1} \mathrm{H}-\mathrm{NMR}$ (DMSO-d6, $500 \mathrm{MHz}$ ): $\delta 8.48$ (s, 1H), $8.26(\mathrm{~d}, \mathrm{~J}=7.9 \mathrm{~Hz}, 1 \mathrm{H}), 8.14-8.05(\mathrm{~m}$, 3H), 8.03 (br, 1H), 7.76 (t, J = 8.2 Hz, 1H), 7.70 (t, J = $7.3 \mathrm{~Hz}, 1 \mathrm{H}), 7.57$ (t, J = $7.9 \mathrm{~Hz}, 2 \mathrm{H}), 7.52$ (br, 1H), $6.21(\mathrm{~s}, 1 \mathrm{H}) \mathrm{ppm} ;{ }^{13} \mathrm{C}\left\{{ }^{1} \mathrm{H}\right\}$-NMR (DMSO-d6, $125 \mathrm{MHz}$ ): $\delta 169.1$ (CO), 164.8 (CO), 147.8 (C), $138.0(\mathrm{C}), 133.9(\mathrm{CH}), 133.8(\mathrm{CH}), 130.3(\mathrm{CH}), 129.6(\mathrm{CH}), 129.0(\mathrm{C}), 128.9(\mathrm{CH}), 123.6(\mathrm{CH}), 121.9$ $(\mathrm{CH}), 74.6(\mathrm{CH}) \mathrm{ppm}$; IR (neat): vmax $\left(\mathrm{cm}^{-1}\right)=3204(\mathrm{~m}), 1715$ (s), 1686 (s), 1539 (s), 1524 (s), 1346 (s), 1331 (s), 1252 (s), 1109 (s), 1024 (s), 712 (s); HRMS (ESI): m/z calculated for $\mathrm{C}_{15} \mathrm{H}_{12} \mathrm{~N}_{2} \mathrm{NaO}_{5}[\mathrm{M}+\mathrm{Na}]^{+}$ 323.0638, found: 323.0627 .<smiles>CC(=O)OC(C(N)=O)c1ccc(Cl)cc1</smiles>

\section{2-amino-1-(4-chlorophenyl)-2-oxoethyl acetate 7c}

Prepared from 6c $(130 \mathrm{mg}, 0.28 \mathrm{mmol})$ according to Procedure D (1 h reaction time). The crude reaction mixture was dried over a stream of nitrogen. Purification: column chromatography on silicagel (cyclohexane/EtOAc 1/1, $\mathrm{R}_{\mathrm{f}}=0.17$ ). Isolated as a white solid. Yield: $49 \mathrm{mg}, 0.216 \mathrm{mmol}, 77 \%$.

m.p.: 111-114 ${ }^{\circ} \mathrm{C} ;{ }^{1} \mathrm{H}-\mathrm{NMR}\left(\mathrm{CDCl}_{3}, 400 \mathrm{MHz}\right): \delta 7.38(\mathrm{~d}, \mathrm{~J}=8.3 \mathrm{~Hz}, 2 \mathrm{H}), 7.34(\mathrm{~d}, \mathrm{~J}=8.3 \mathrm{~Hz}, 2 \mathrm{H}), 6.33$ (br, 1H), 6.27 (br, 1H), $6.02(\mathrm{~s}, 1 \mathrm{H}), 2.18(\mathrm{~s}, 3 \mathrm{H}) \mathrm{ppm} ;{ }^{13} \mathrm{C}\left\{{ }^{1} \mathrm{H}\right\}-\mathrm{NMR}\left(\mathrm{CDCl}_{3}, 125 \mathrm{MHz}\right): \delta 170.8(\mathrm{CO})$, $169.2(\mathrm{CO}), 135.2(\mathrm{C}), 133.8(\mathrm{C}), 129.1(\mathrm{CH}), 128.9(\mathrm{CH}), 74.6(\mathrm{CH}), 21.1\left(\mathrm{CH}_{3}\right) \mathrm{ppm}$; IR (neat): vmax $\left(\mathrm{cm}^{-1}\right)=3311(\mathrm{~m}), 3178(\mathrm{~m}), 2967(\mathrm{~s}), 1735(\mathrm{~m}), 1632(\mathrm{w}), 1488(\mathrm{~s}), 1409(\mathrm{~m}), 1367(\mathrm{~s}), 1222(\mathrm{w}), 1093$ (s), $1039(\mathrm{~m}), 821(\mathrm{~s}), 661(\mathrm{w}), 544(\mathrm{~m})$; HRMS (ESI): $\mathrm{m} / \mathrm{z}$ calculated for $\mathrm{C}_{10} \mathrm{H}_{10} \mathrm{ClNO}_{3}[\mathrm{M}+\mathrm{Na}]^{+}$ 250.0241, found: 250.0246 . 


\section{2-hydroxy-4-methyl- $N$-tritylpentanamide 5a}<smiles>CC(C)CC(O)C(=O)NC(c1ccccc1)(c1ccccc1)c1ccccc1</smiles>

Isolated from experiment $\mathrm{E} 4$ in the mechanistic section (cyclohexane/EtOAc 6/1, $\mathrm{R}_{\mathrm{f}}=0.15$ in cyclohexane/EtOAc 1:1). Isolated as a white solid.

m.p.: 171-172 ${ }^{\circ} \mathrm{C} ;{ }^{1} \mathrm{H}-\mathrm{NMR}\left(\mathrm{CDCl}_{3}, 500 \mathrm{MHz}\right): \delta 7.77$ (br, 1H), 7.36-7.25 (m, 9H), 7.21 (d, J = 7.0 Hz, $6 \mathrm{H}), 4.13(\mathrm{dd}, \mathrm{J}=9.5,2.3 \mathrm{~Hz}, 1 \mathrm{H}), 2.77$ (br, 1H), 1.88-1.77 (m, 1H), 1.71-1.54 (m, 2H), 0.97 (d, J = 6.6 $\mathrm{Hz}, 3 \mathrm{H}), 0.93(\mathrm{~d}, \mathrm{~J}=6.6 \mathrm{~Hz}, 3 \mathrm{H}) \mathrm{ppm} ;{ }^{13} \mathrm{C}\left\{{ }^{1} \mathrm{H}\right\}-\mathrm{NMR}\left(\mathrm{CDCl}_{3}, 125 \mathrm{MHz}\right): \delta 173.2(\mathrm{CO}), 144.7(\mathrm{C}), 128.7$ $(\mathrm{CH}), 128.1(\mathrm{CH}), 127.2(\mathrm{CH}), 71.3(\mathrm{CH}), 70.0(\mathrm{C}), 43.9\left(\mathrm{CH}_{2}\right), 24.7(\mathrm{CH}), 23.6\left(\mathrm{CH}_{3}\right), 21.4\left(\mathrm{CH}_{3}\right) \mathrm{ppm}$; IR (neat): vmax $\left(\mathrm{cm}^{-1}\right)=3238(\mathrm{w}), 1664(\mathrm{~s}), 1654(\mathrm{~s}), 1500$ (s), 752 (s), 696 (s), 628 (m); HRMS (ESI): $m / z$ calculated for $\mathrm{C}_{25} \mathrm{H}_{28} \mathrm{NO}_{2}[\mathrm{M}+\mathrm{H}]^{+}$374.2115, found: 374.2108 . 


\section{Copies of spectra}

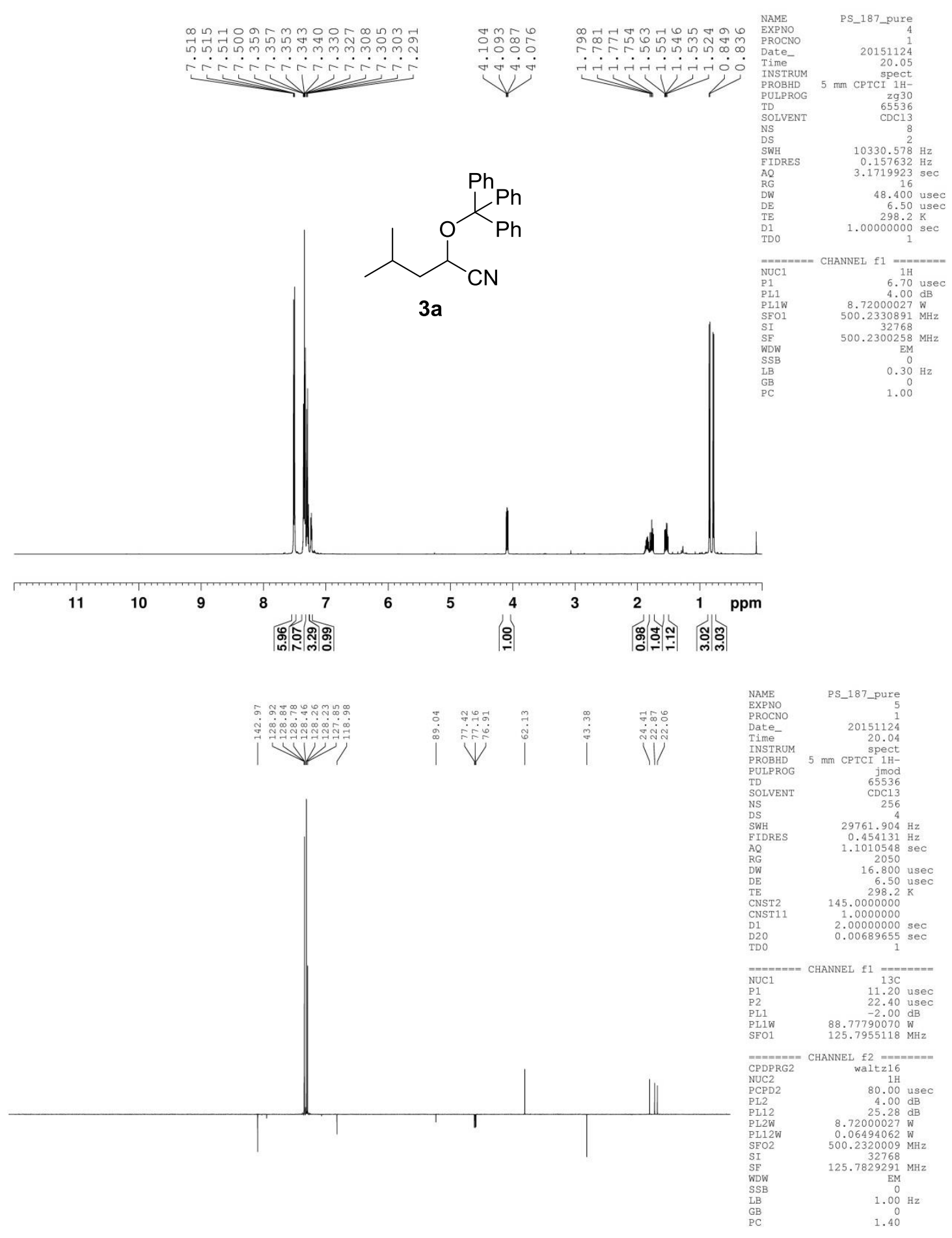

\begin{tabular}{|c|c|c|c|c|c|c|c|c|c|}
\hline 200 & 180 & 160 & 140 & 120 & 100 & 80 & 60 & 40 & ppm \\
\hline
\end{tabular}



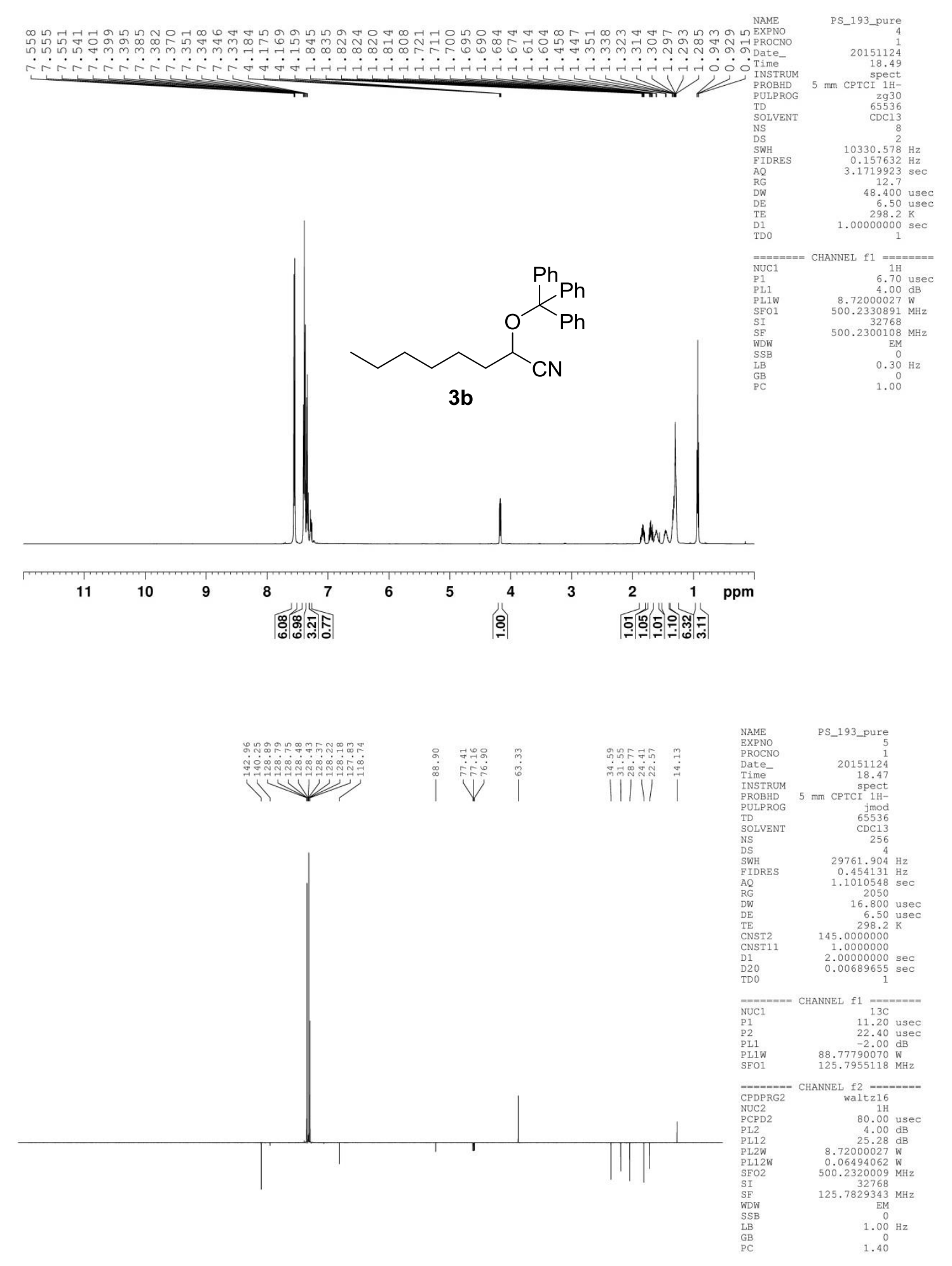

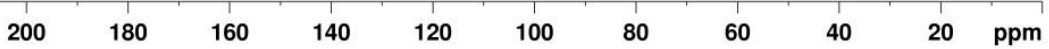



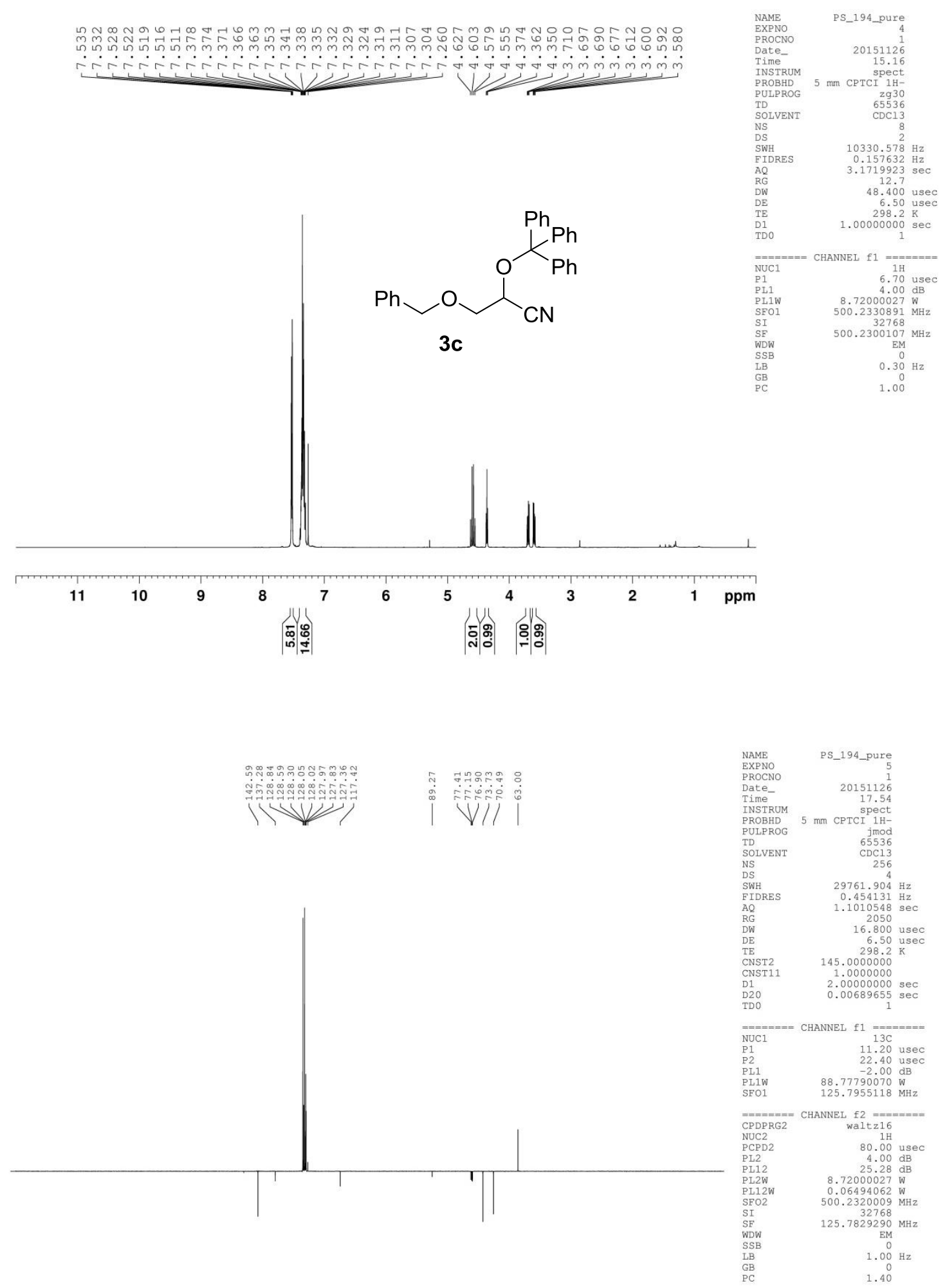

200

$\begin{array}{lll}180 & 160 \quad 140\end{array}$

120

100

80

$60 \quad 40$ 

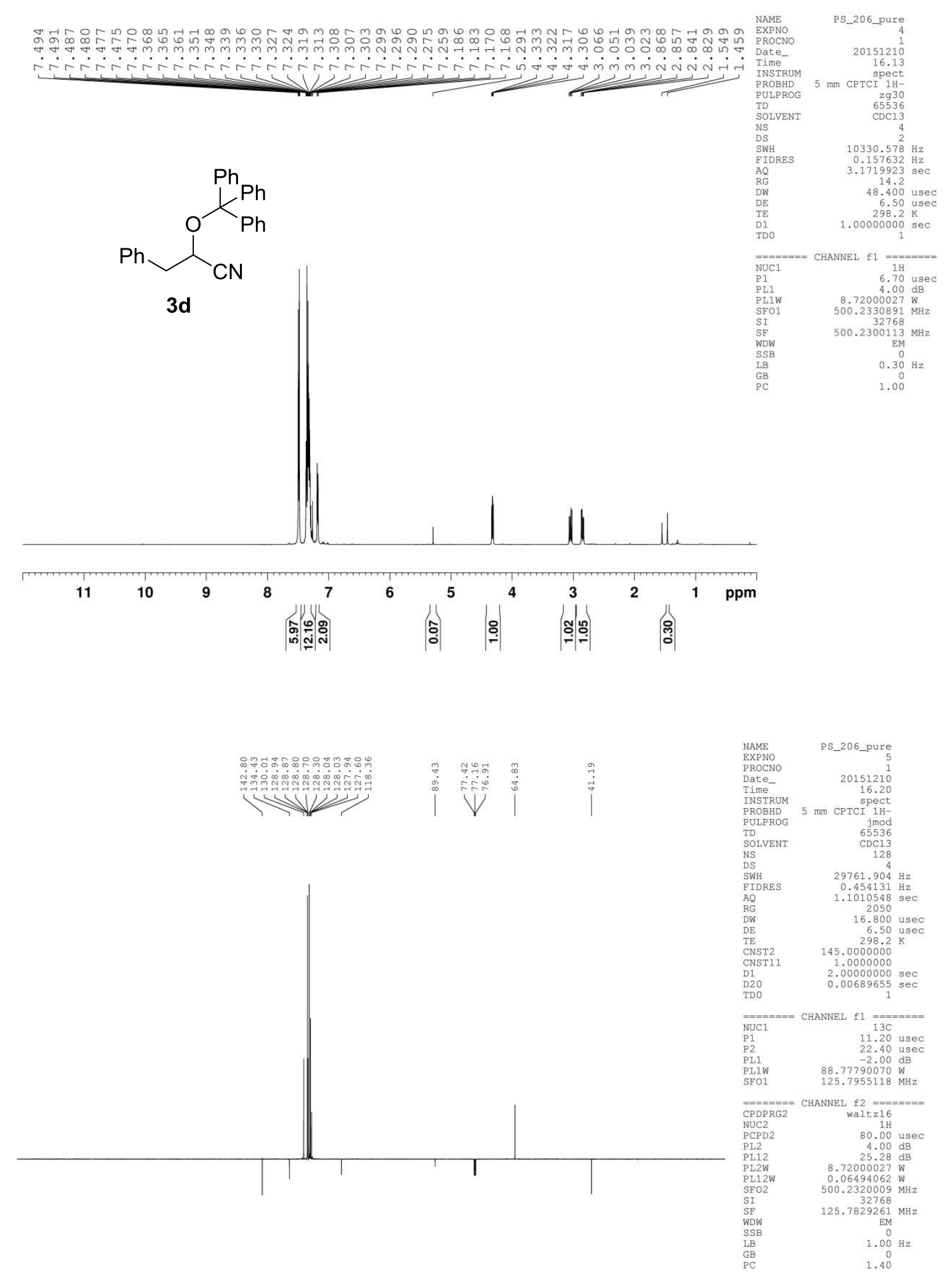

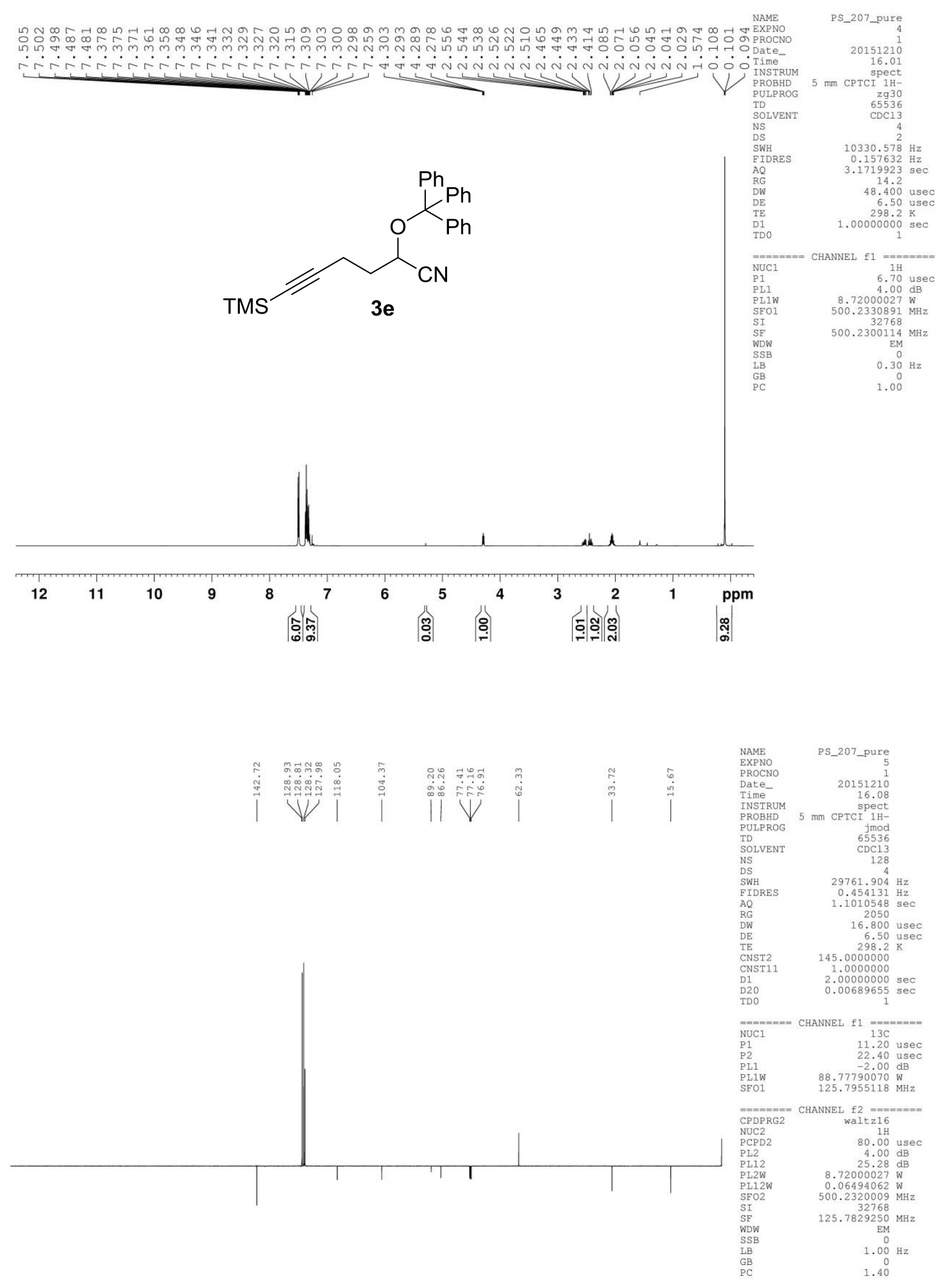

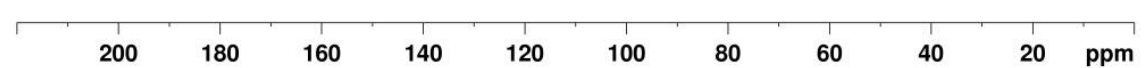



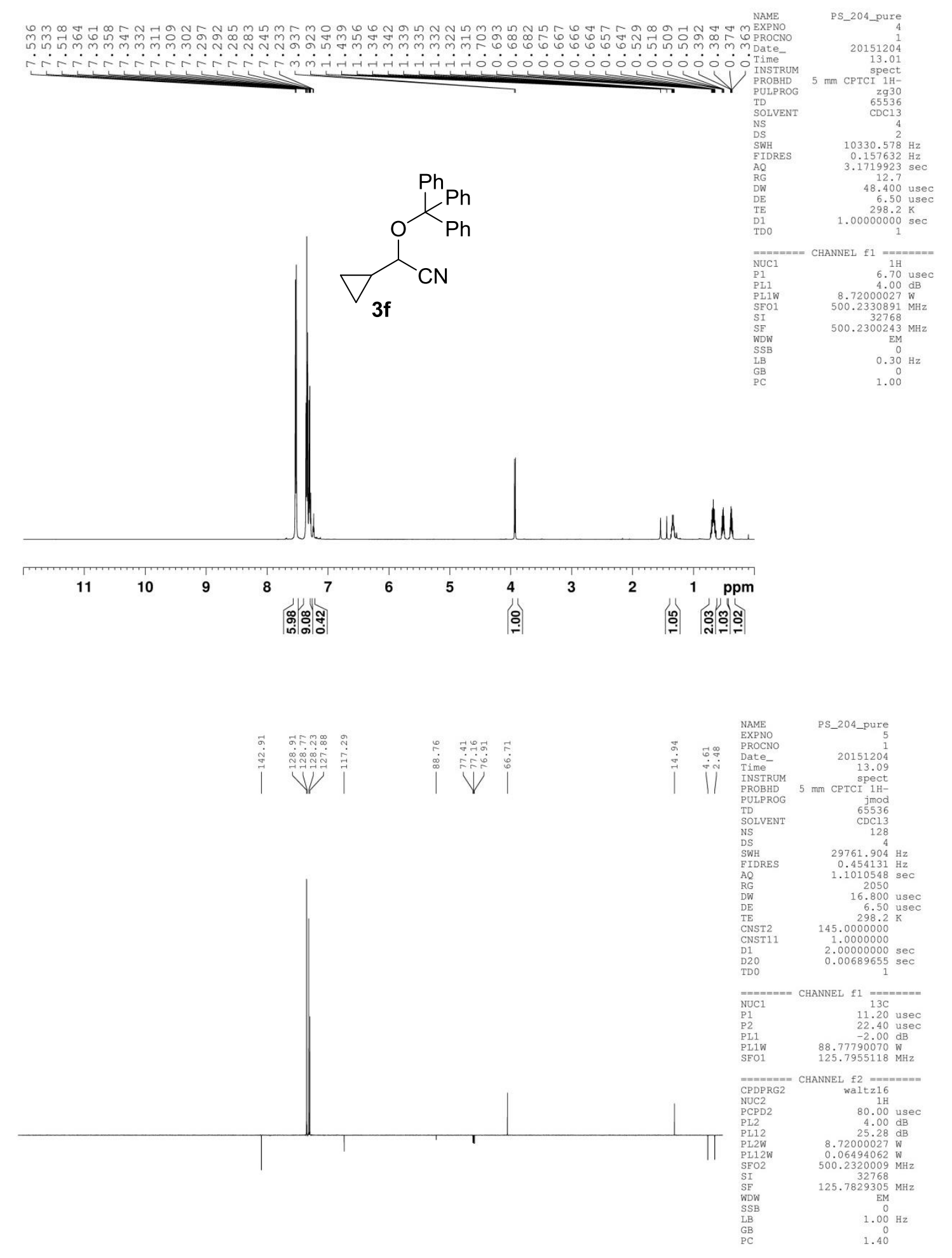

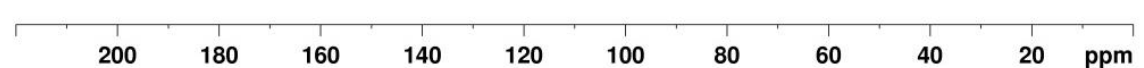



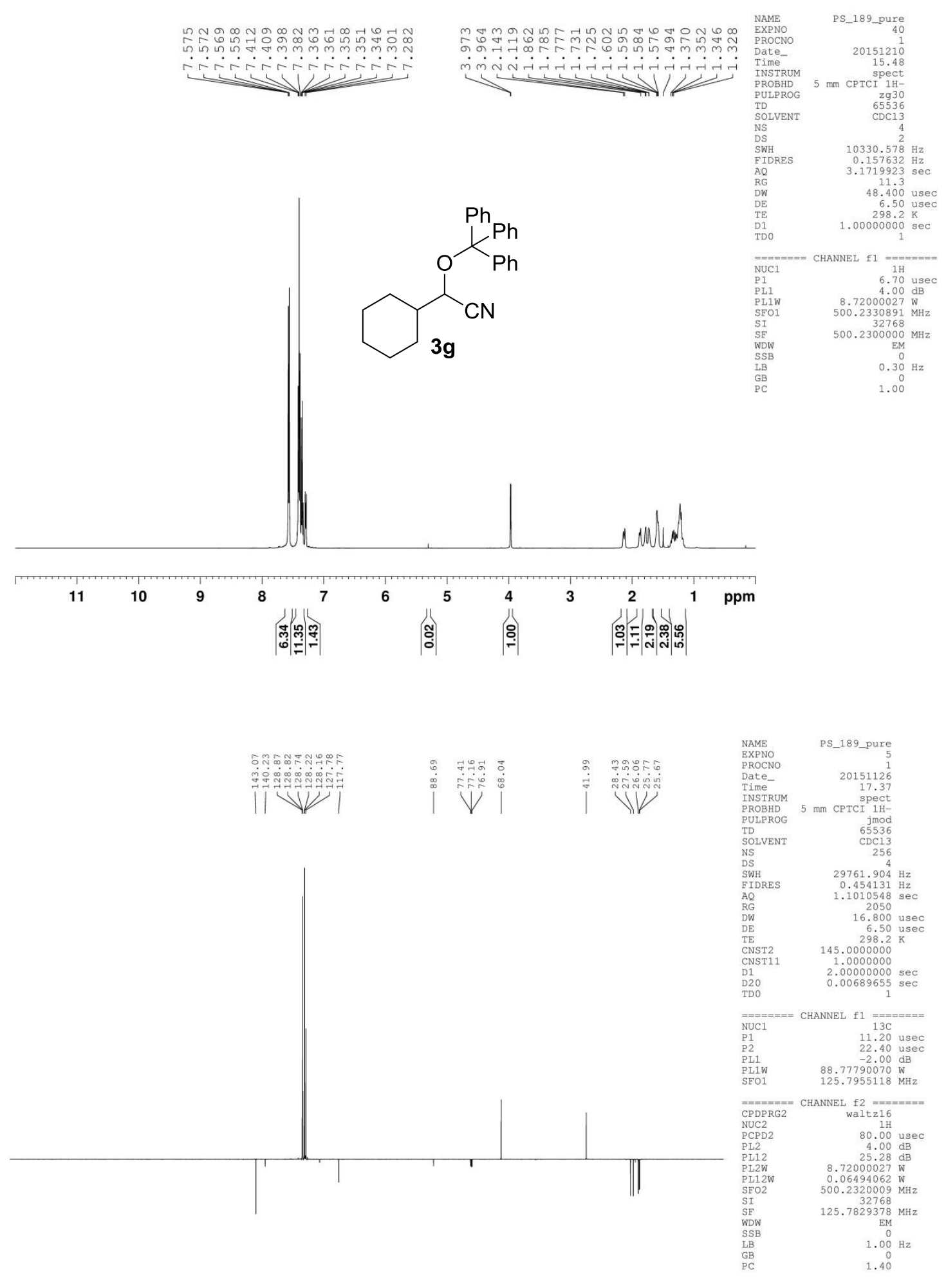

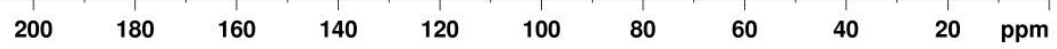



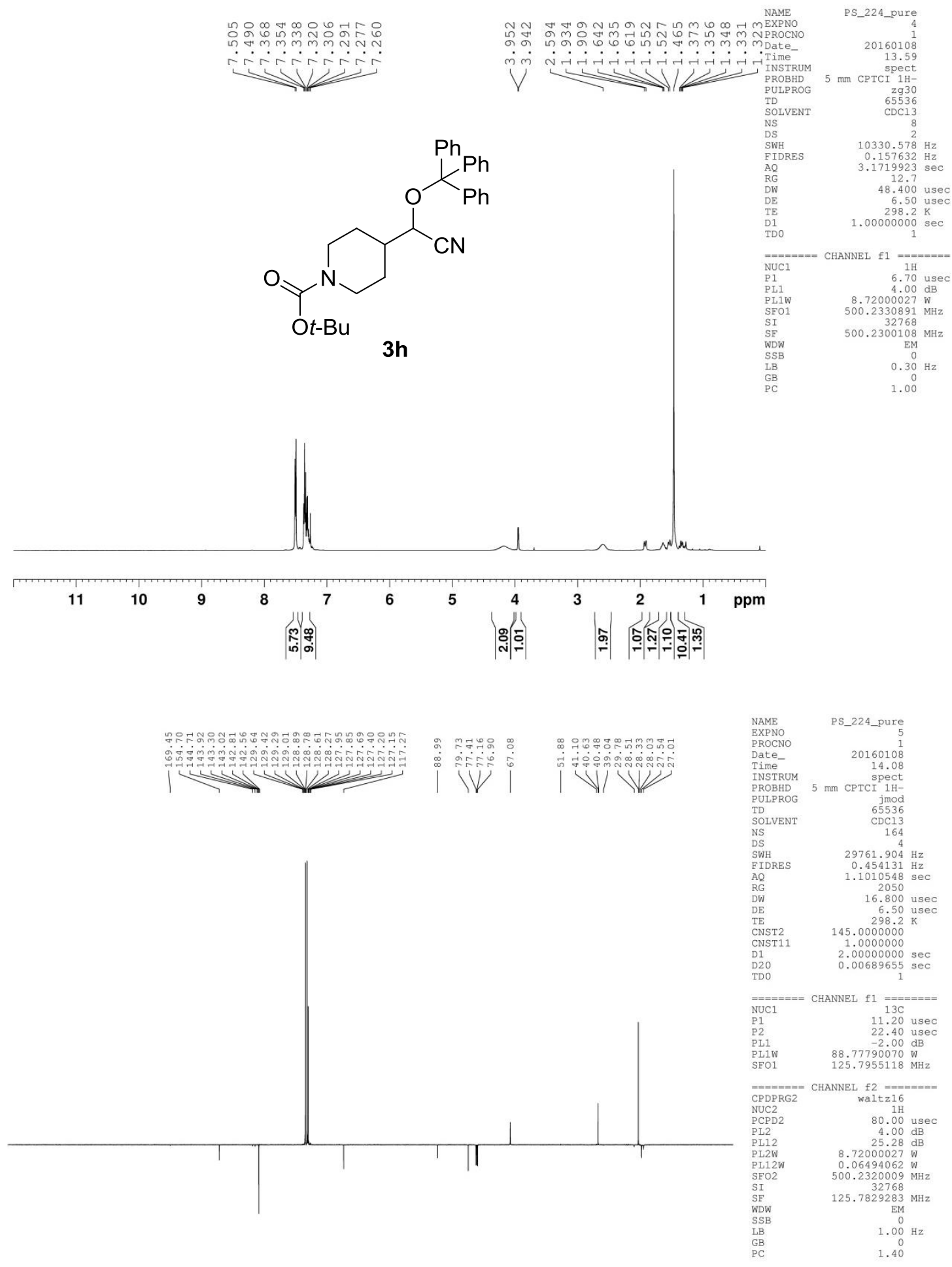

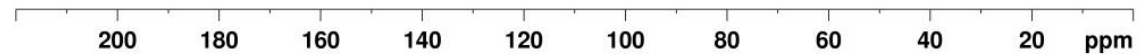




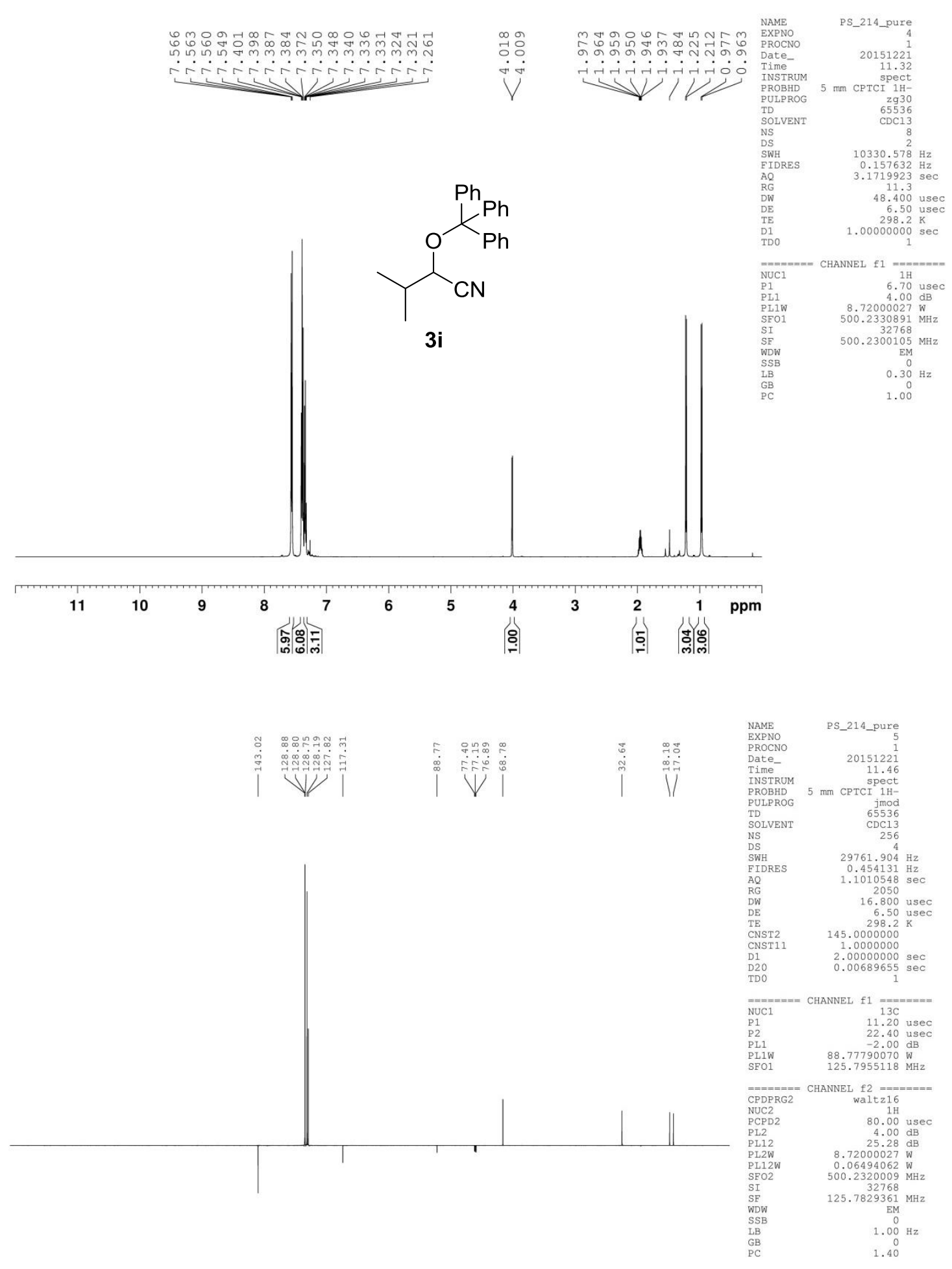

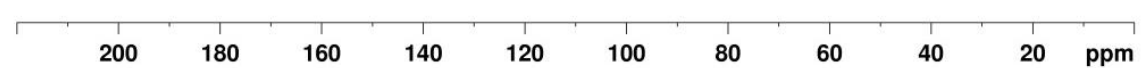




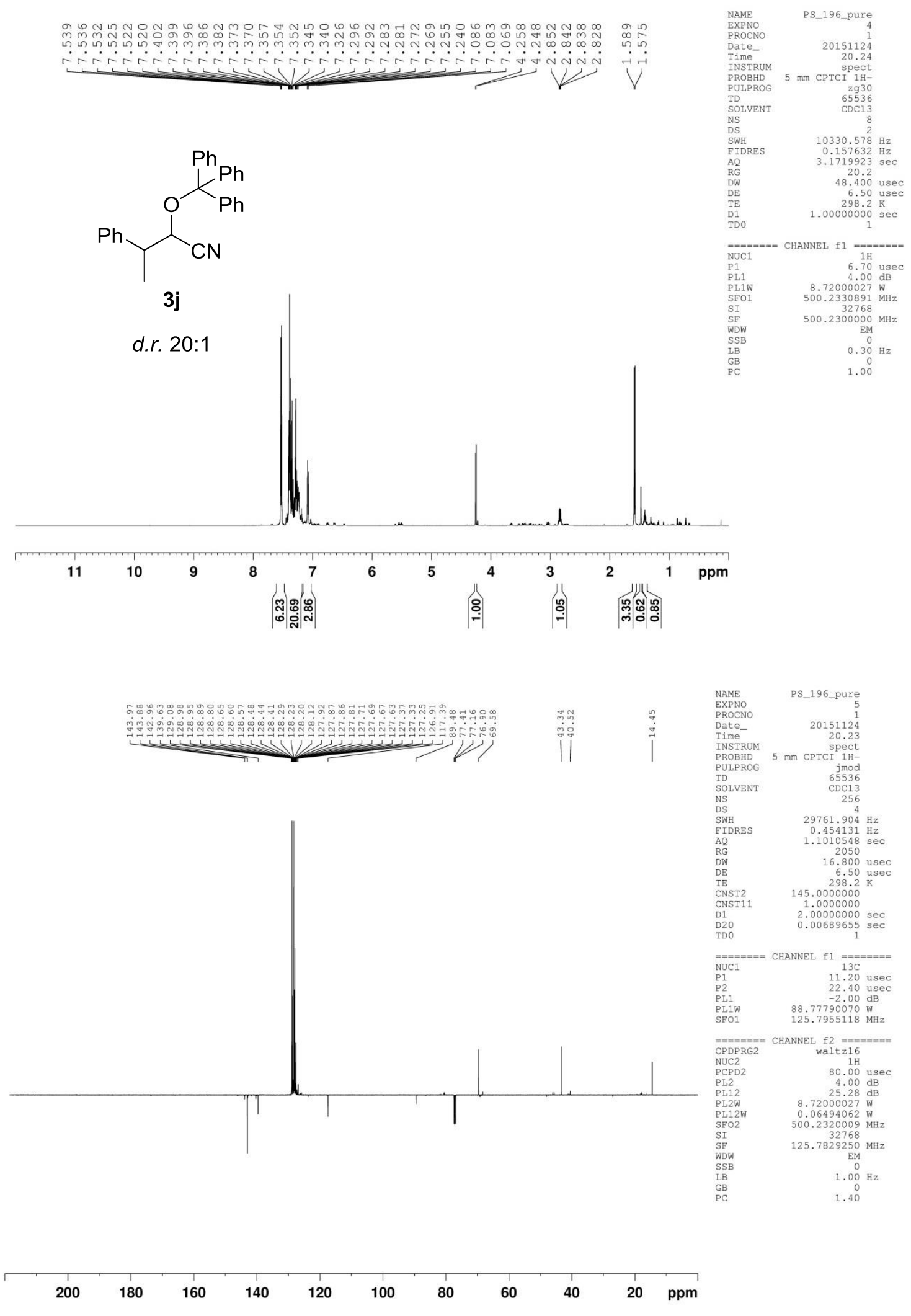



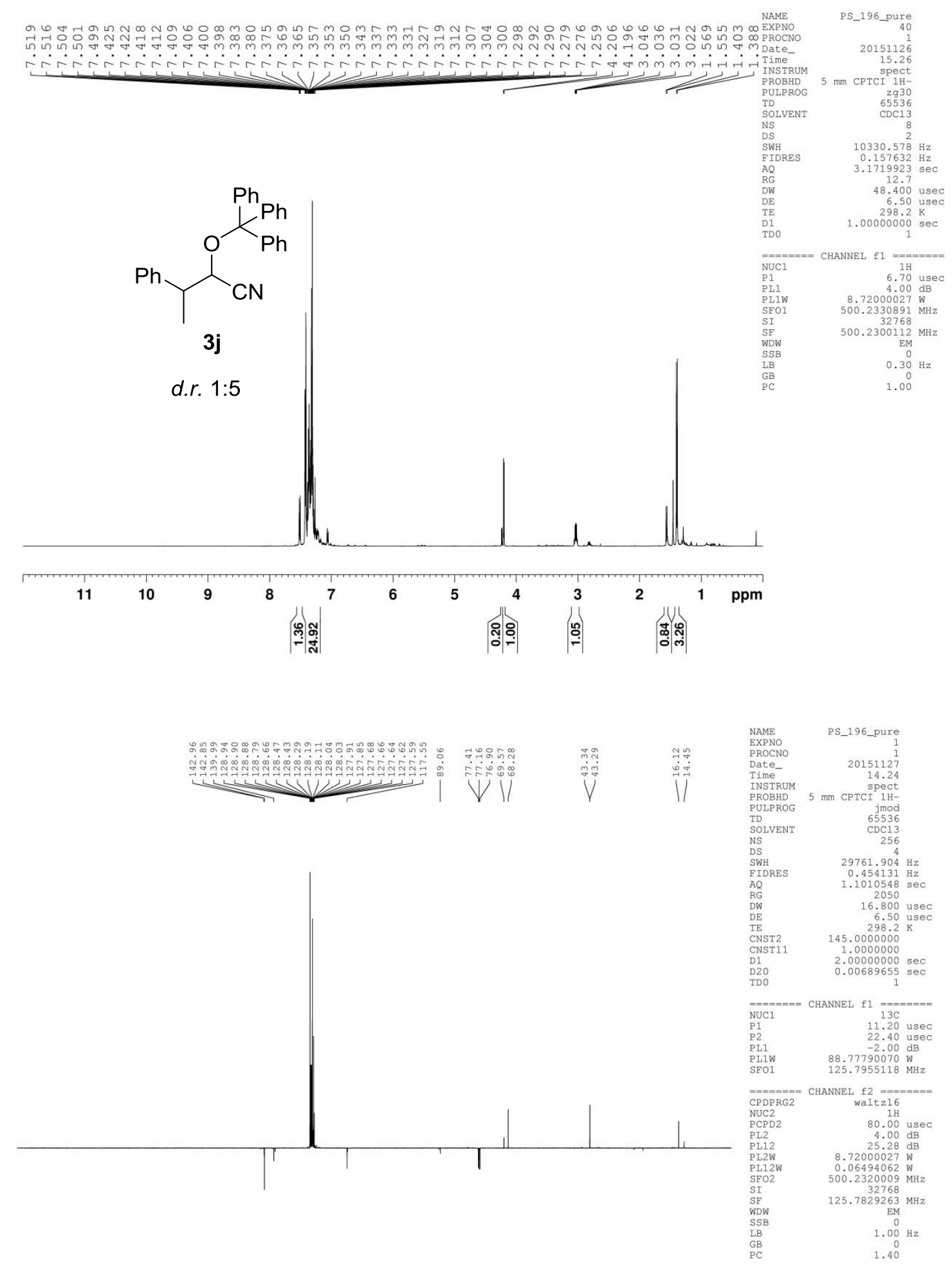

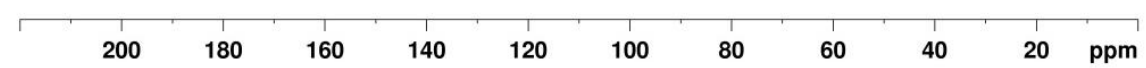



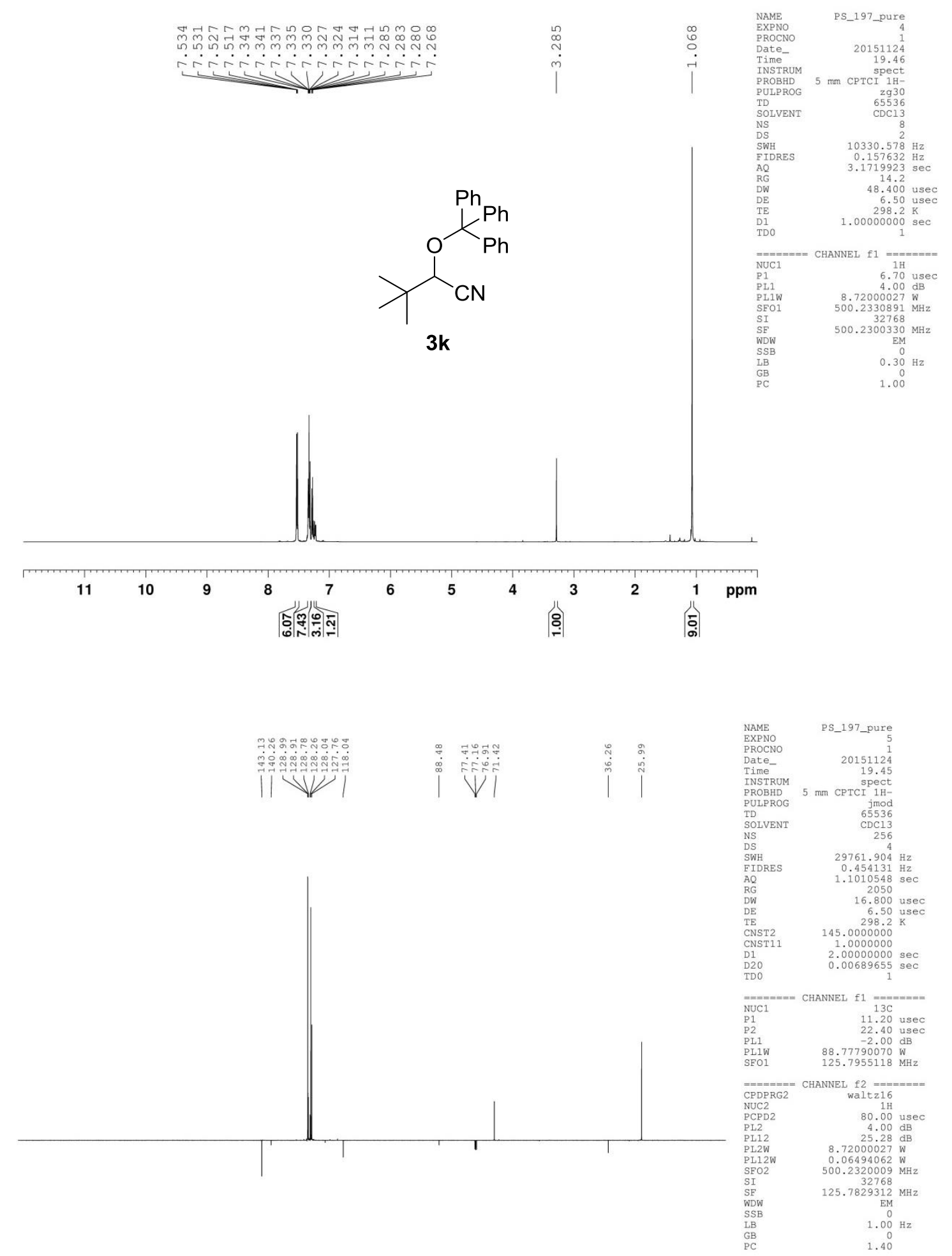

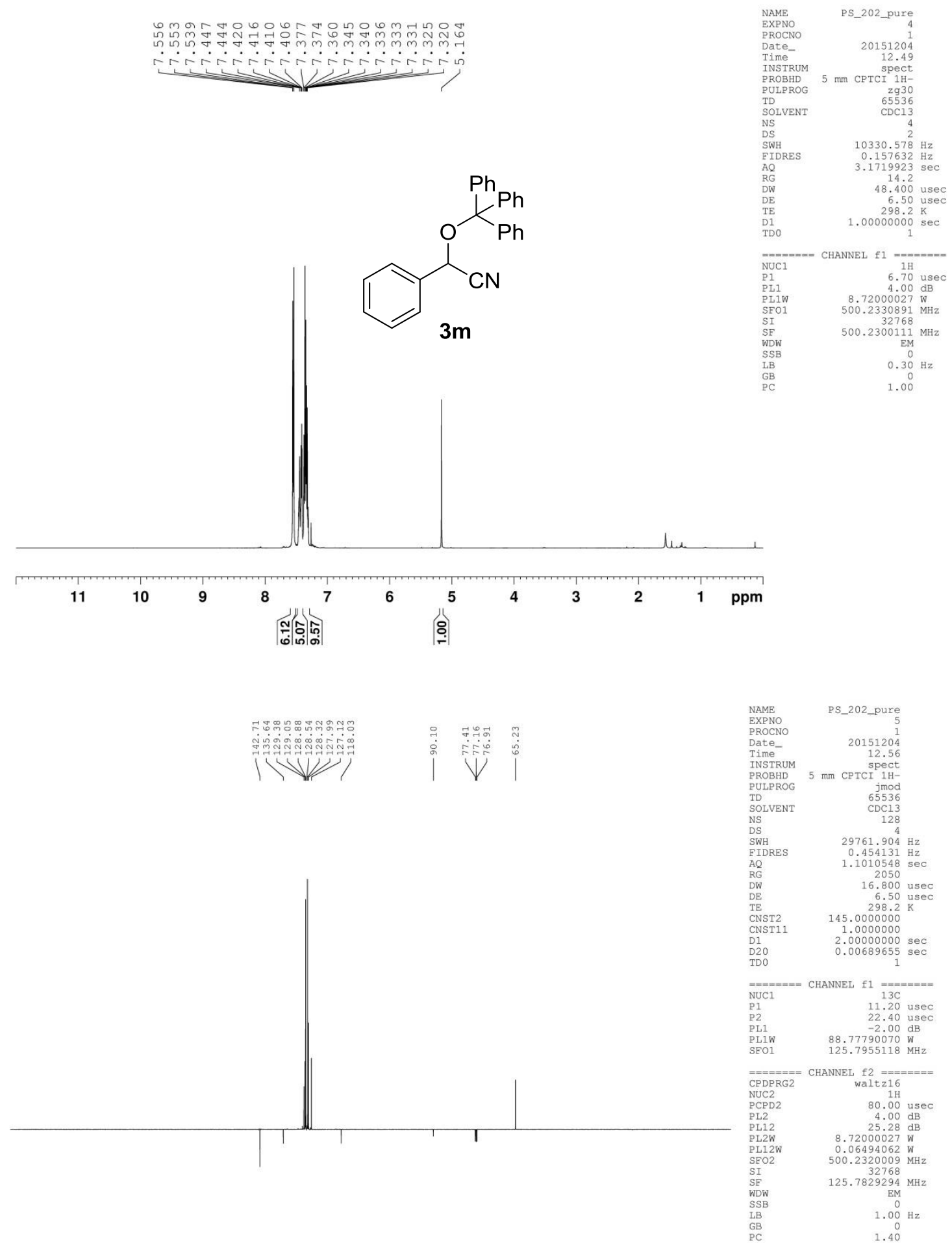

$\begin{array}{lllllllllll}200 & 180 & 160 & 140 & 120 & 100 & 80 & 60 & 40 & 20 & \text { ppm }\end{array}$




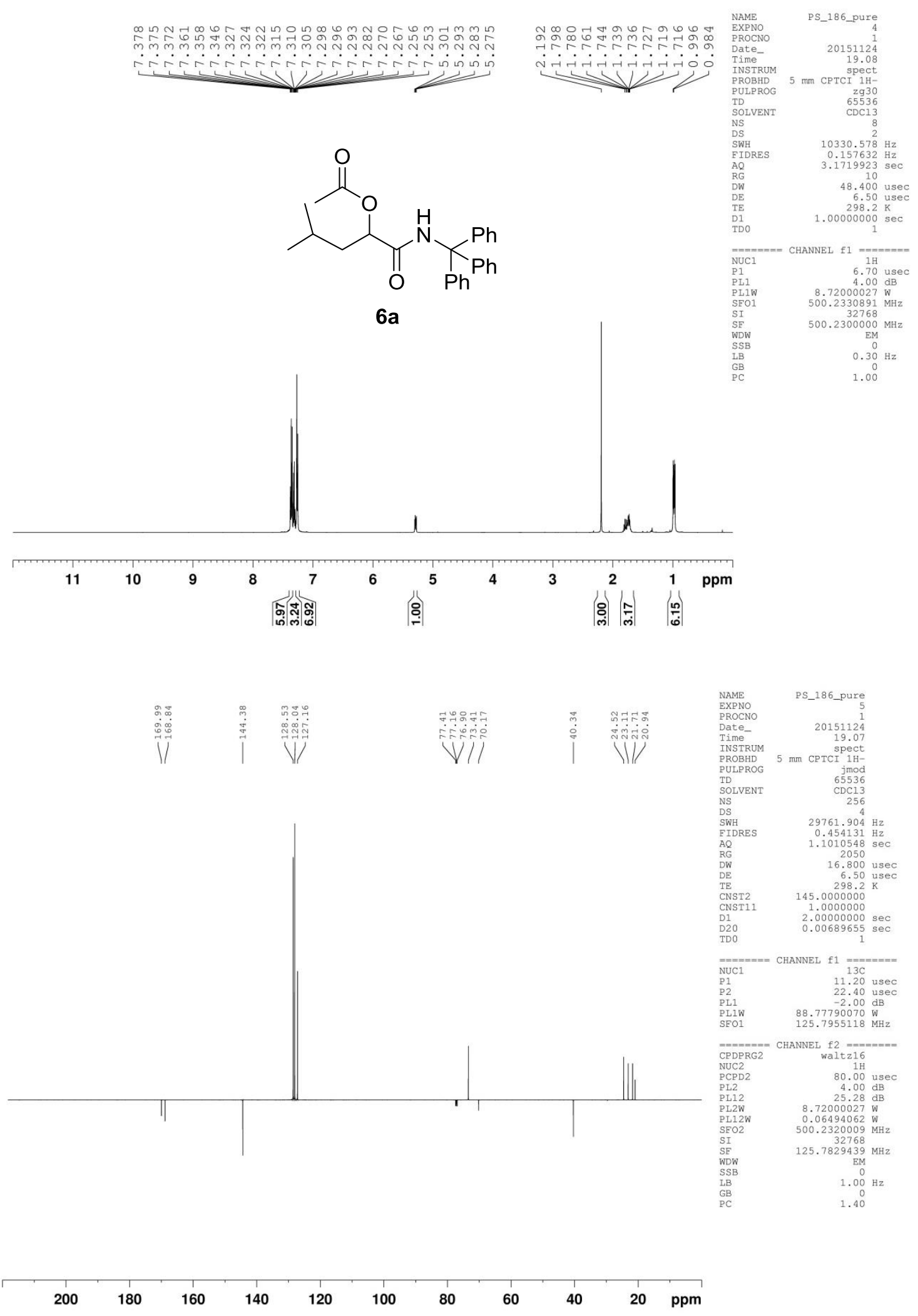




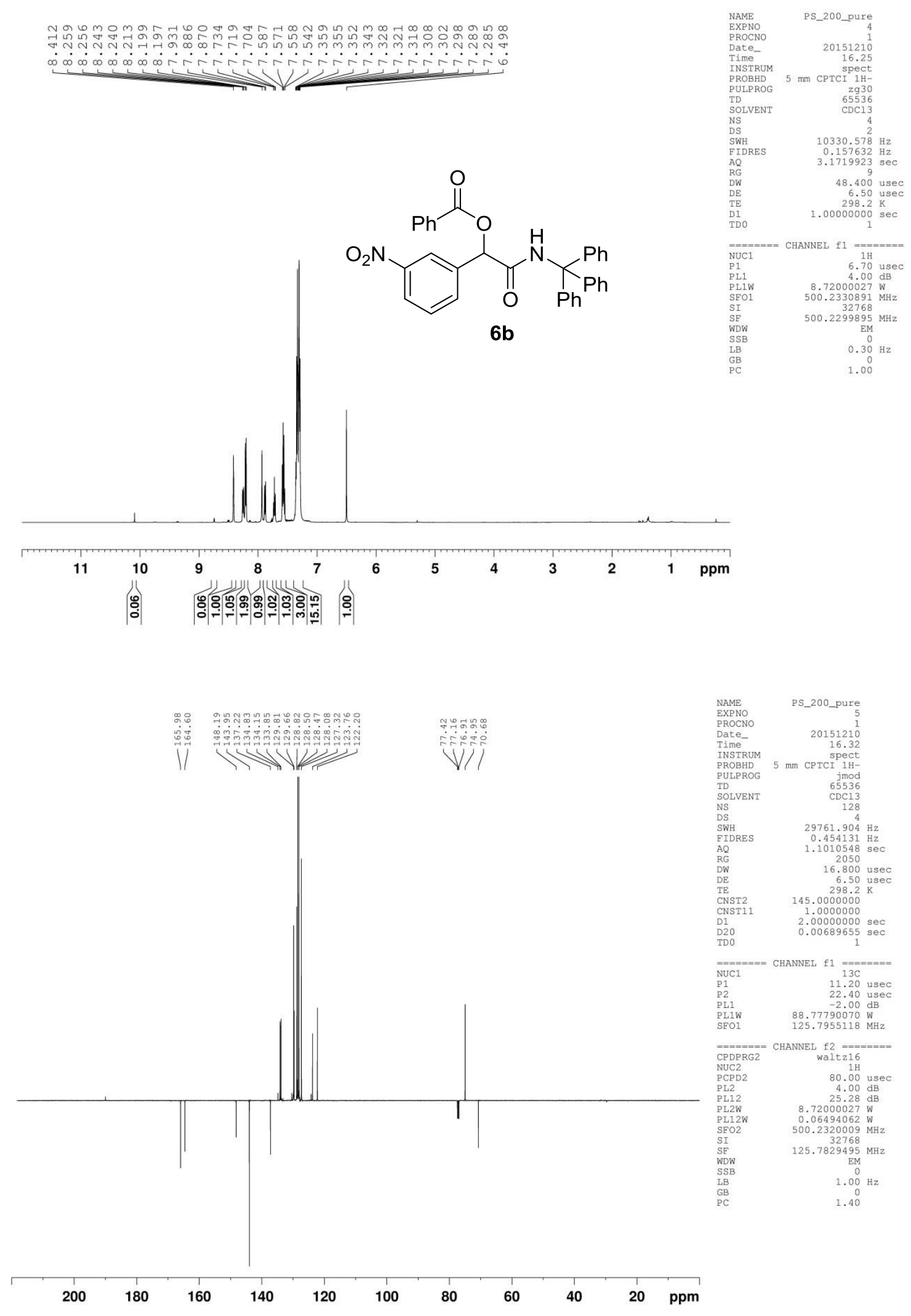



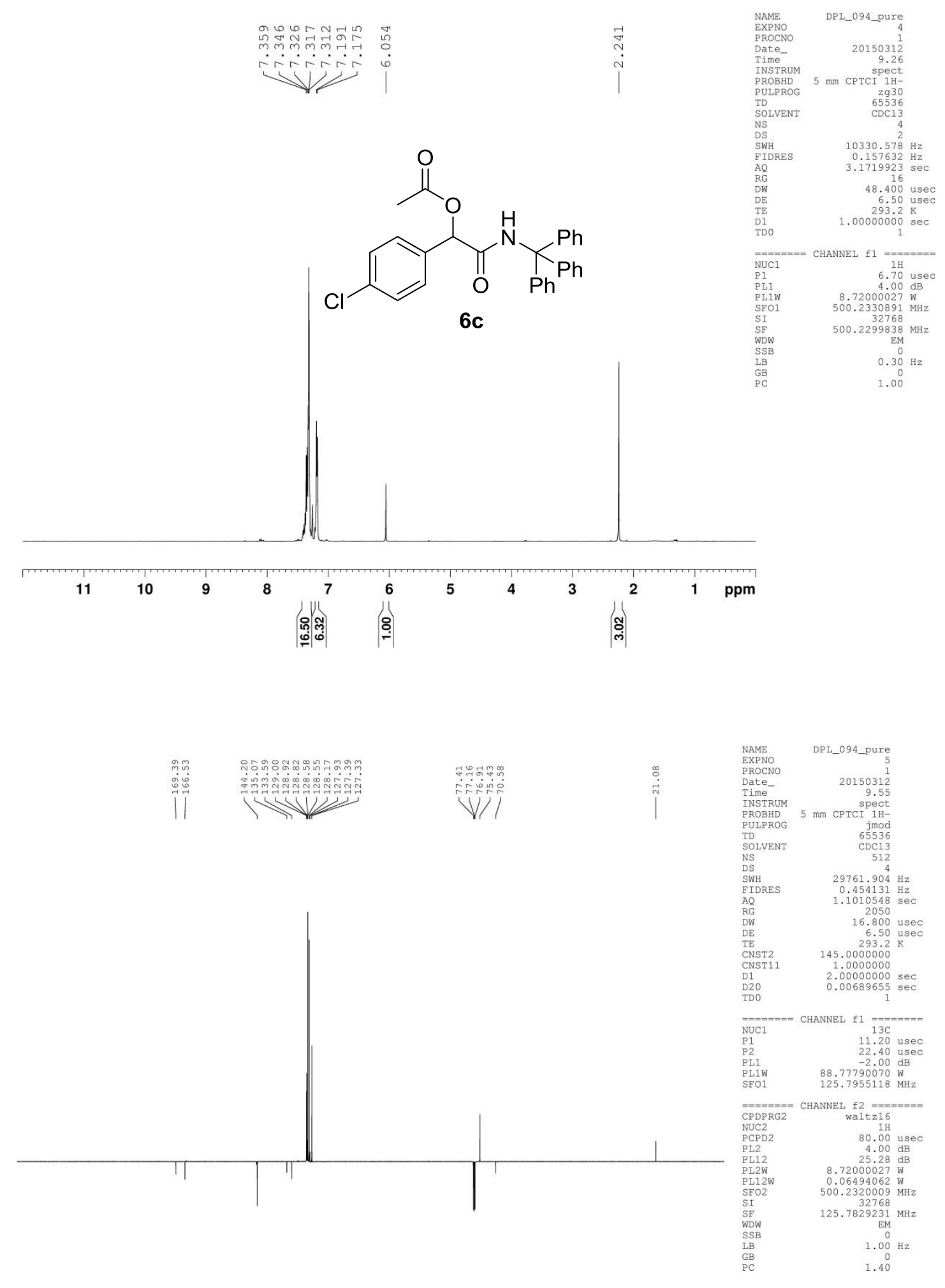

$\begin{array}{lllllllllll}200 & 180 & 160 & 140 & 120 & 100 & 80 & 60 & 40 & 20 & \text { ppm }\end{array}$



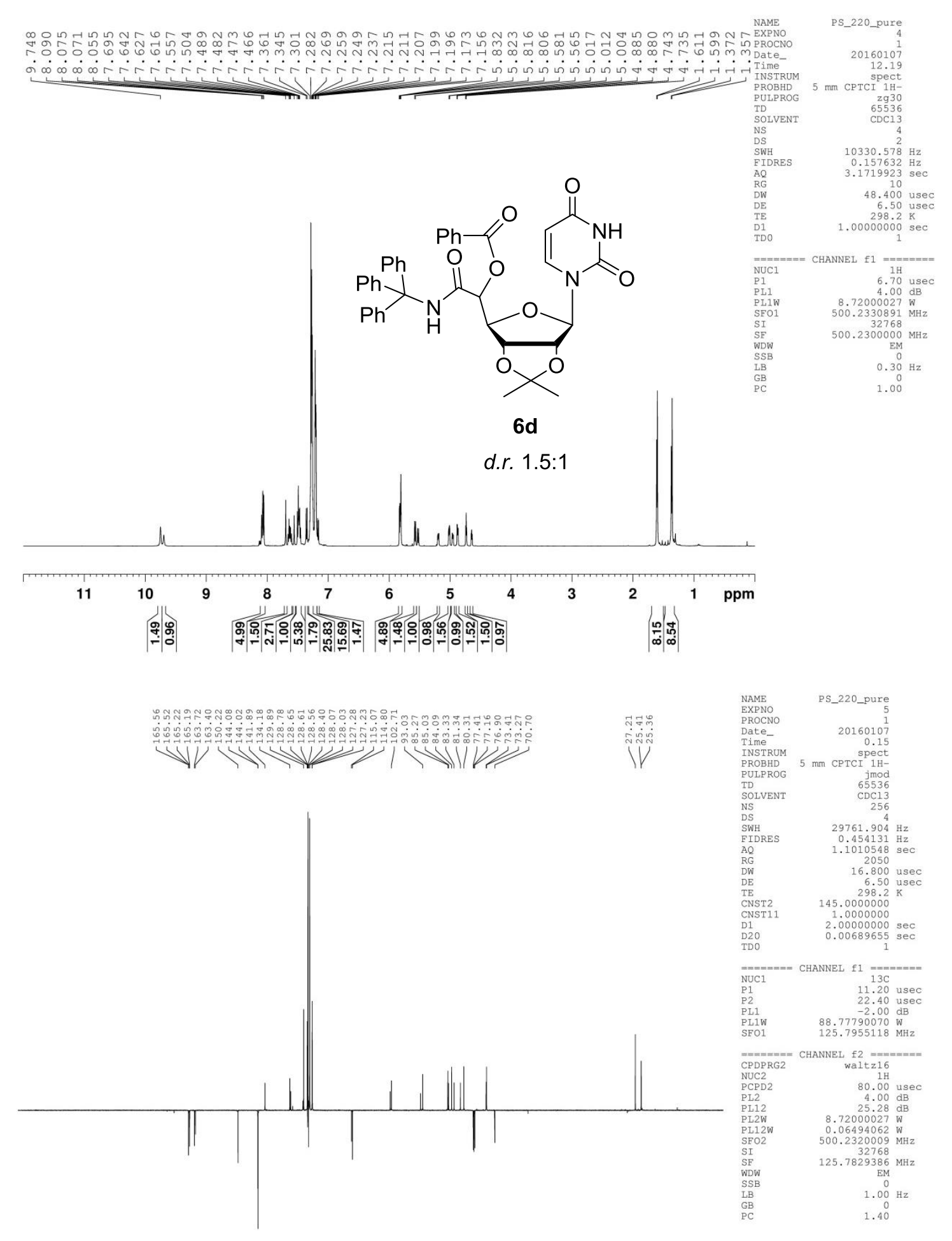

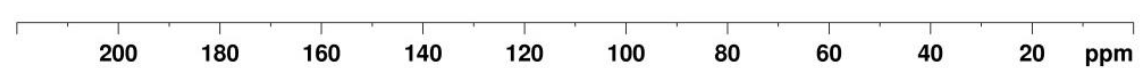




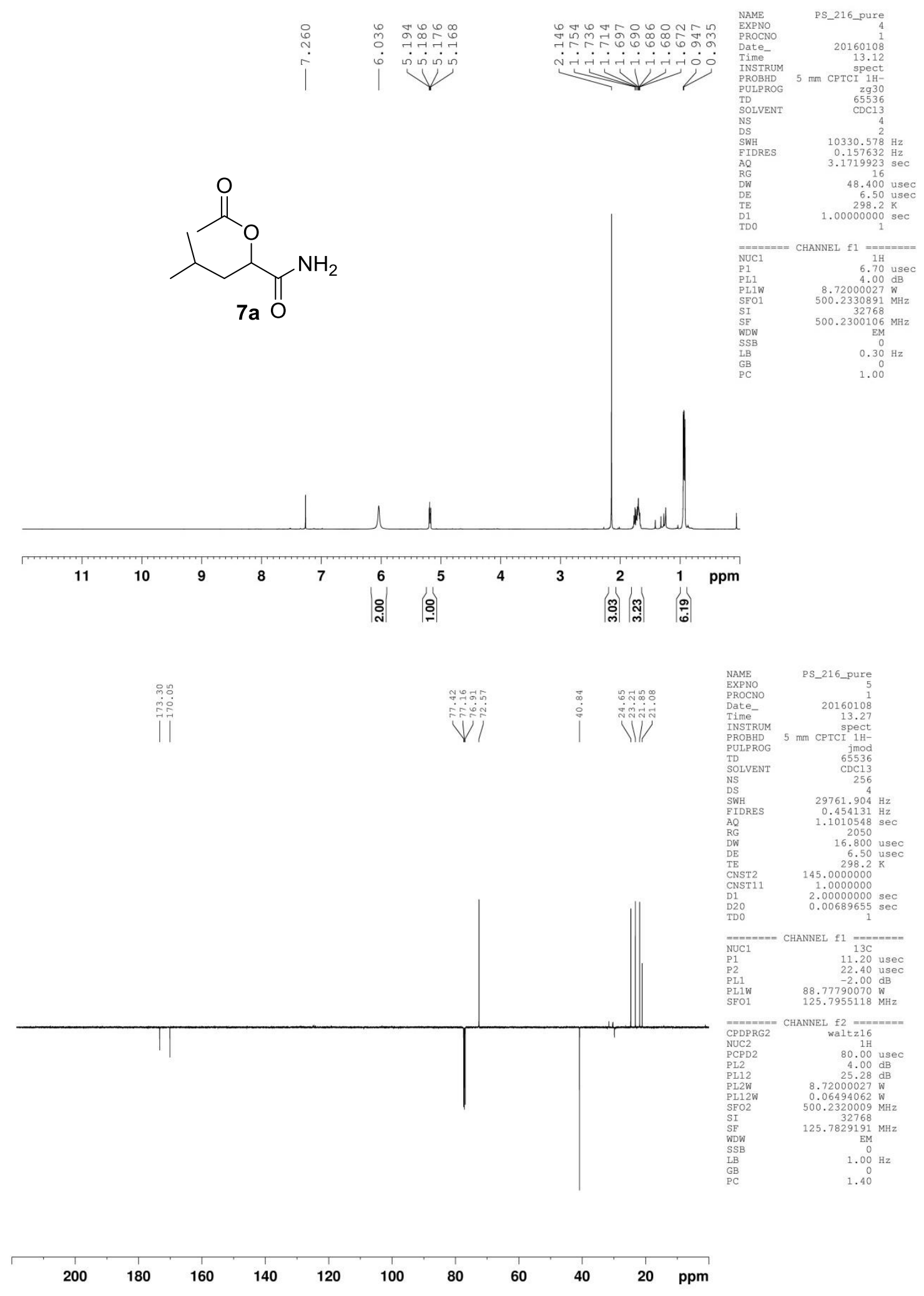



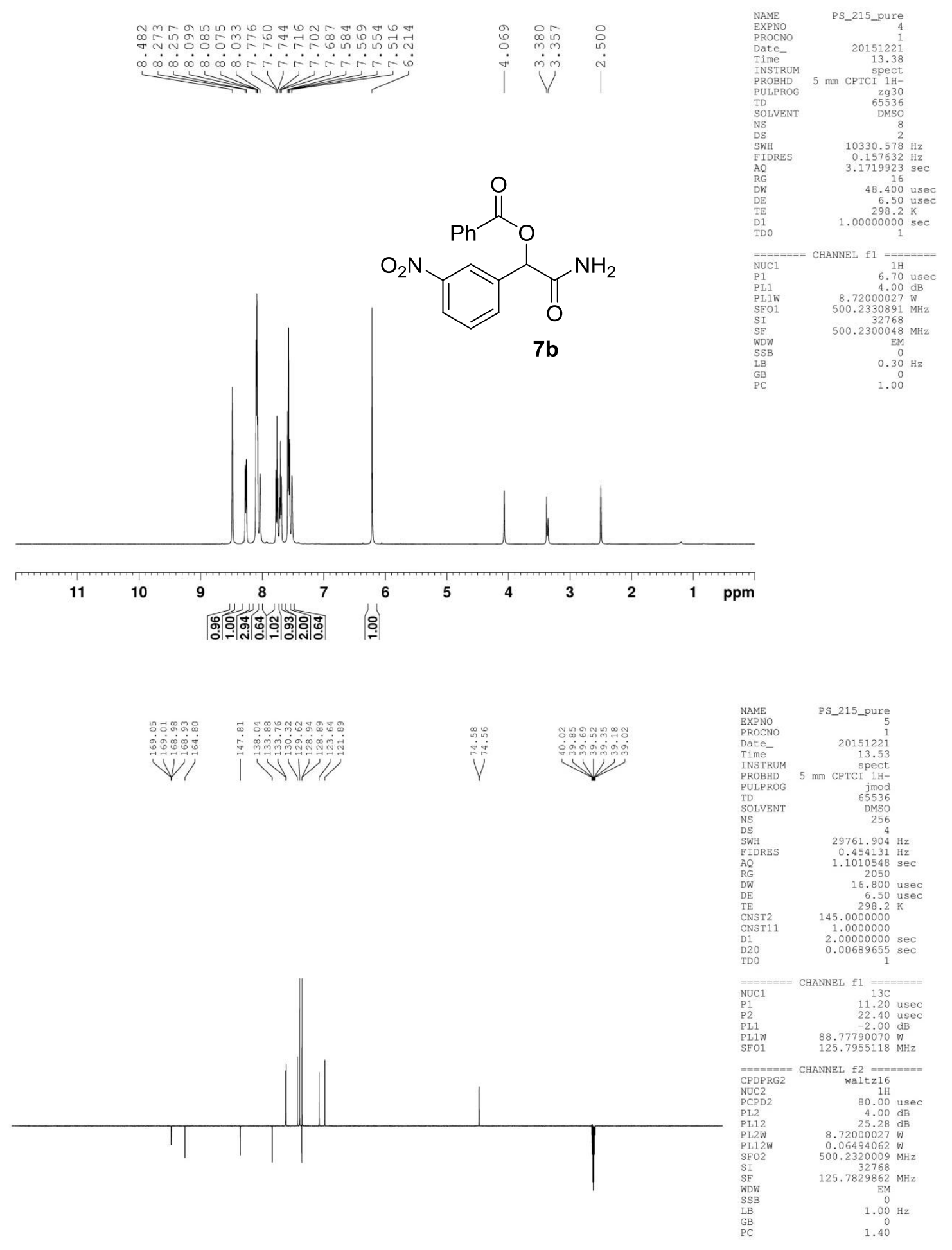

\begin{tabular}{rrrrrrrrrrr}
\hline 200 & 180 & 160 & 140 & 120 & 100 & 80 & 60 & 40 & 20 & $\mathrm{ppm}$ \\
\hline
\end{tabular}



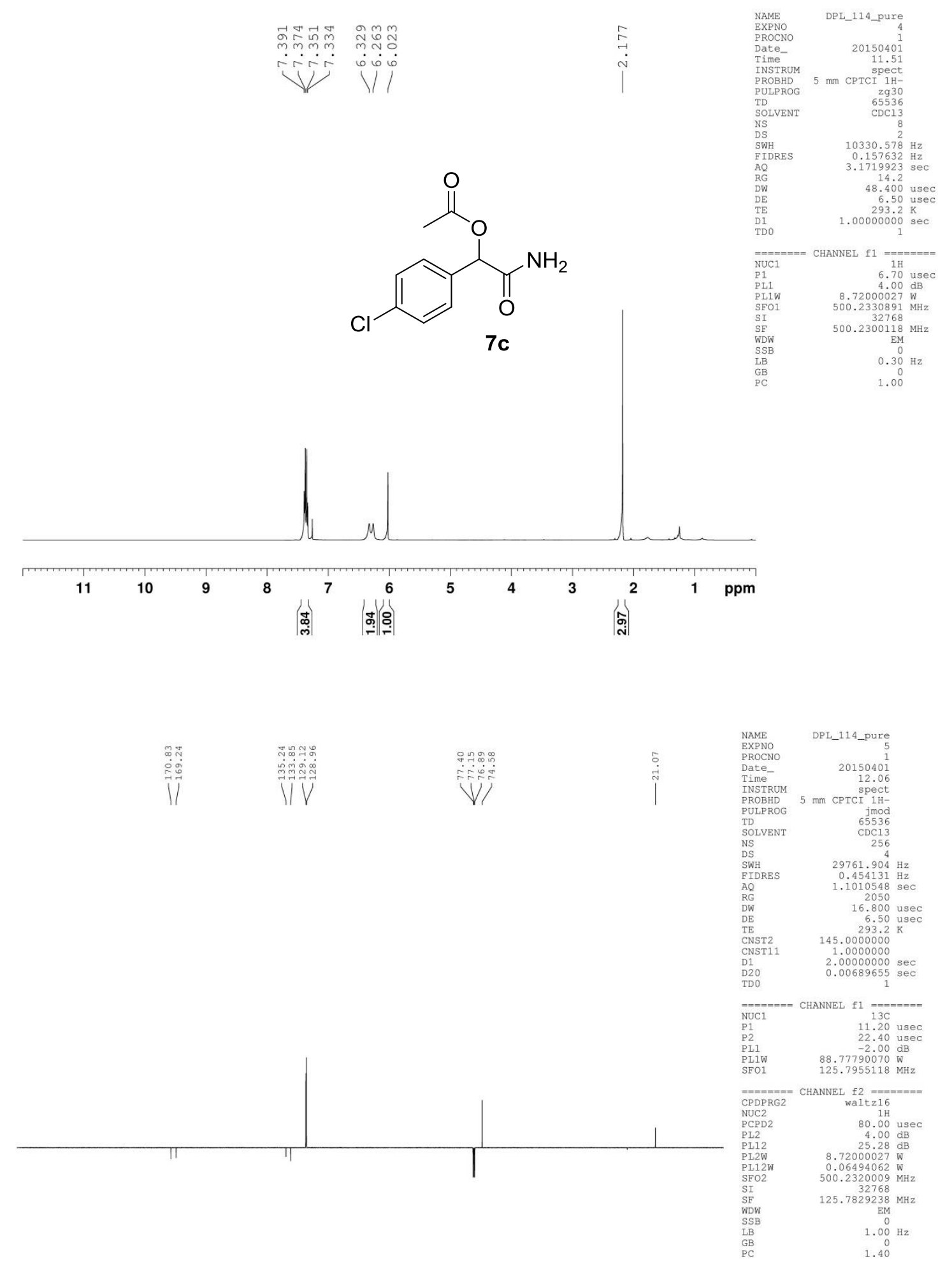

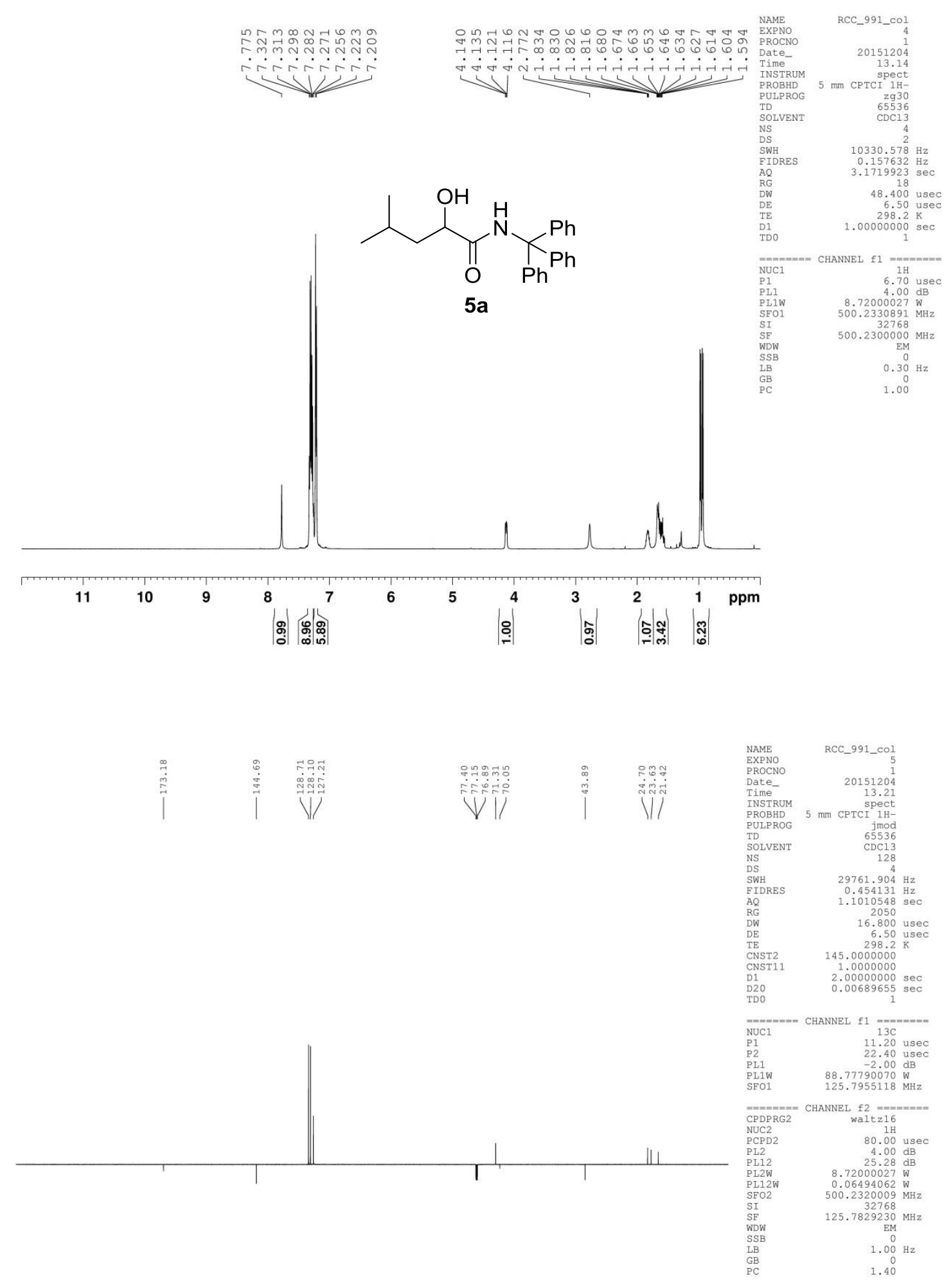

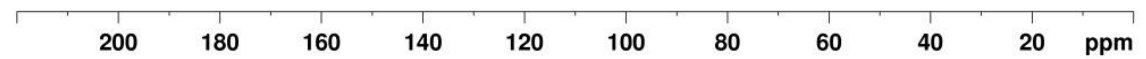


HPLC traces

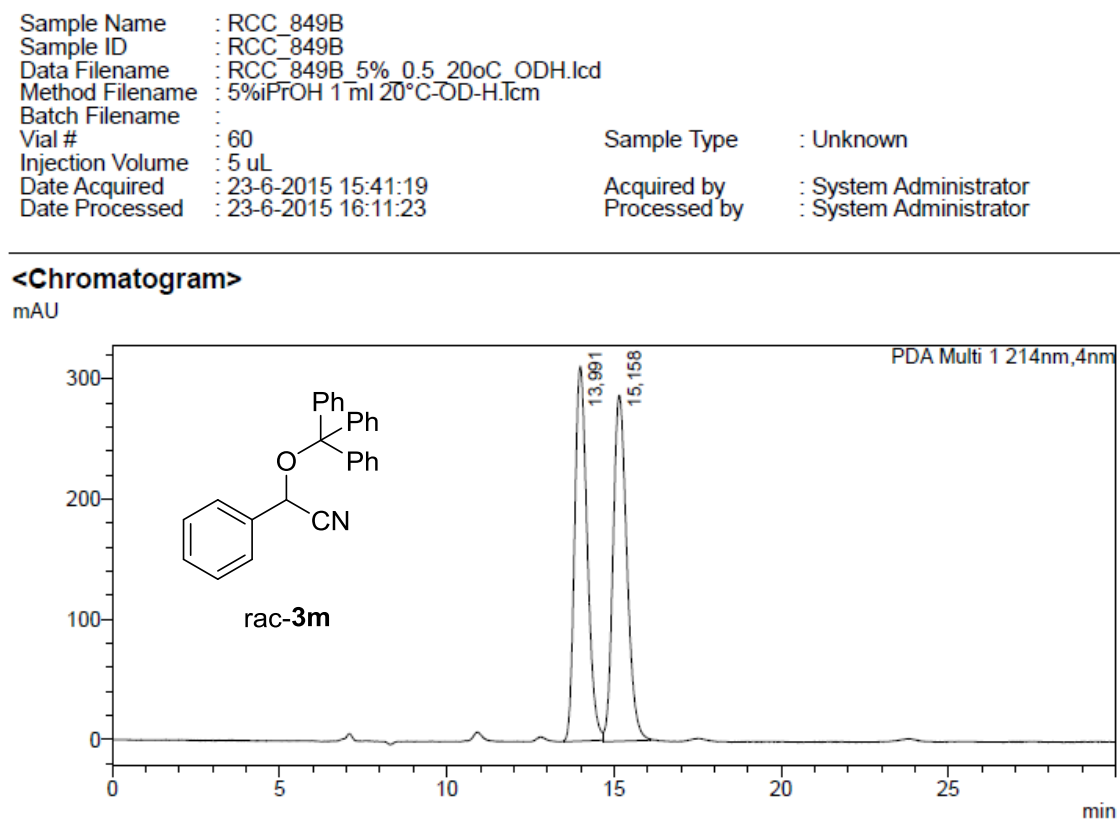

<Peak Table>

PDA Ch1 $214 \mathrm{~nm}$
\begin{tabular}{|r|r|r|}
\hline Ret. Time & \multicolumn{1}{|c|}{ Area } & \multicolumn{1}{|c|}{ Area $\%$} \\
\hline 13,991 & 8125892 & 49,572 \\
\hline 15,158 & 8266157 & 50,428 \\
\hline & 16392049 & 100,000 \\
\hline
\end{tabular}
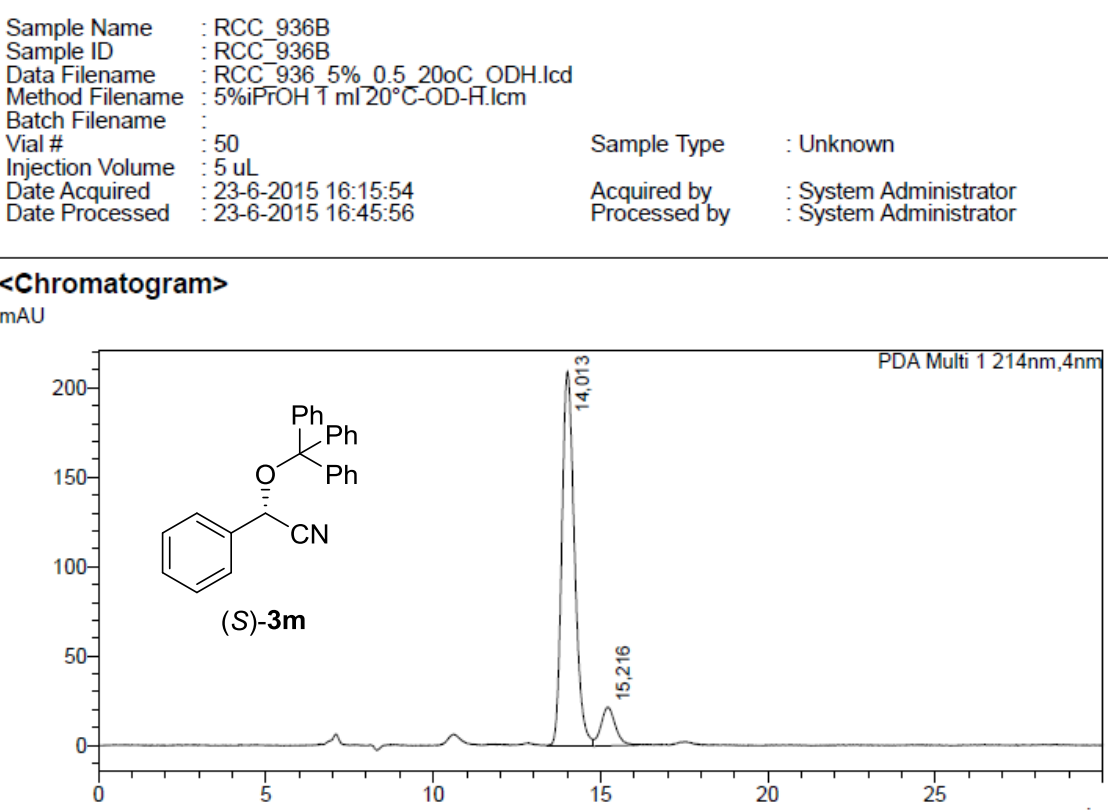

$<$ Peak Table>

PDA Ch1 214nm
\begin{tabular}{|r|r|r|}
\hline Ret. Time & \multicolumn{1}{|c|}{ Area } & \multicolumn{1}{c|}{ Area $\%$} \\
\hline 14,013 & 5503131 & 89,404 \\
\hline 15,216 & 652214 & 10,596 \\
\hline & 6155346 & 100,000 \\
\hline
\end{tabular}




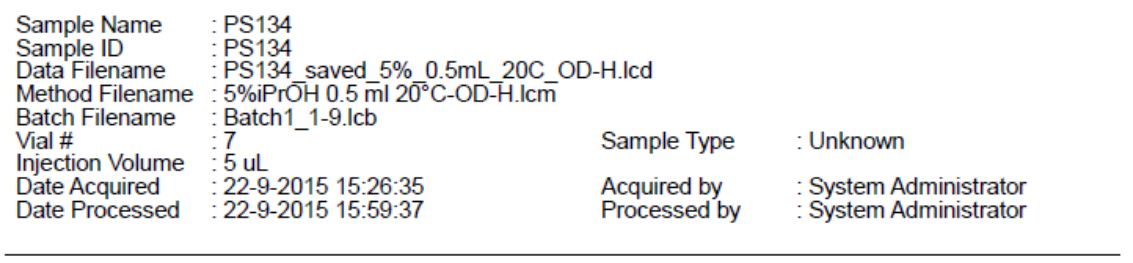

$<$ Chromatogram >

mAU

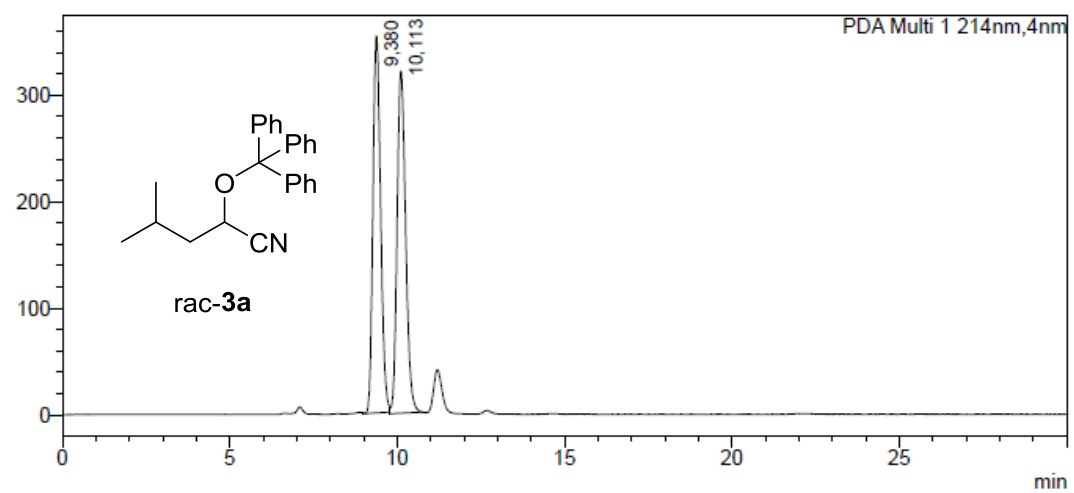

<Peak Table>

PDA Ch1 $214 \mathrm{~nm}$
\begin{tabular}{|r|r|r|}
\hline Ret. Time & Area & \multicolumn{1}{|c|}{ Area $\%$} \\
\hline 9,380 & 5644010 & 49,716 \\
\hline 10,113 & 5708533 & 50,284 \\
\hline & 11352543 & 100,000 \\
\hline
\end{tabular}

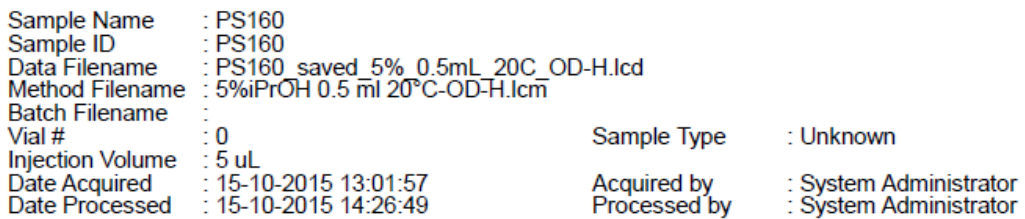

\section{<Chromatogram>}

mAU

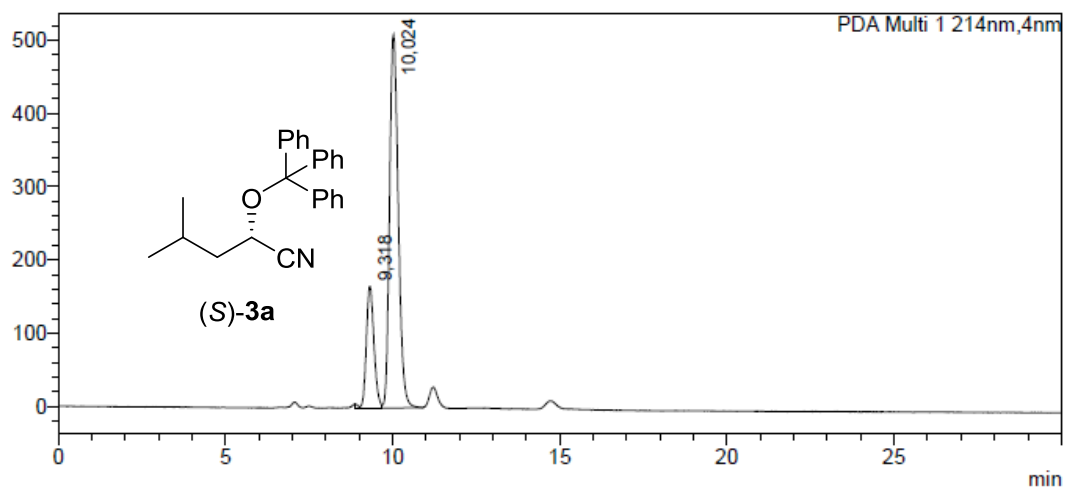

<Peak Table>

PDA Ch1 214nm

\begin{tabular}{|r|r|r|}
\hline Ret. Time & Area & \multicolumn{1}{|c|}{ Area $\%$} \\
\hline 9,318 & 2649685 & 22,303 \\
\hline 10,024 & 9230844 & 77,697 \\
\hline & 11880530 & 100,000 \\
\hline
\end{tabular}




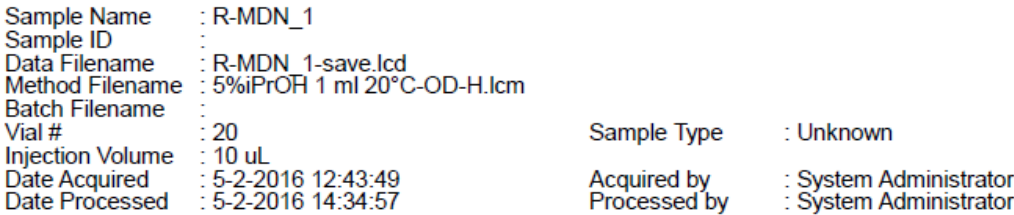

\section{$<$ Chromatogram>}

$\mathrm{mAU}$

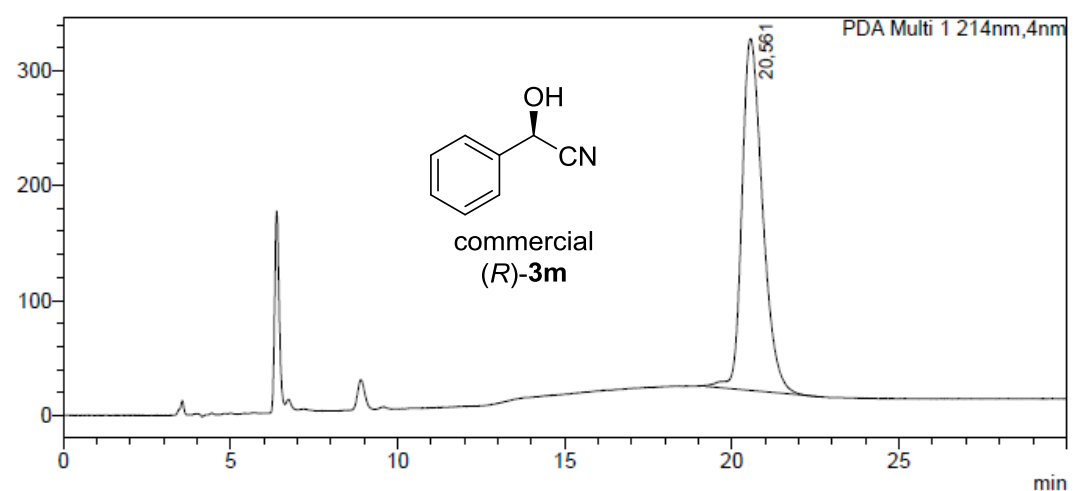

<Peak Table>

PDA Ch1 214nm

\begin{tabular}{|l|l|l}
\hline Ret. Time & Area & Area\% \\
\hline
\end{tabular}

\begin{tabular}{l|r|r|}
\hline 20,561 & 13155812 & 100,000 \\
\hline
\end{tabular}

\begin{tabular}{l|l}
13155812 & 100,000 \\
\hline
\end{tabular}

Sample Name : RCC 945B

Sample ID : RCC_945B

Data Filename : RCC 945B 5\% $120 \mathrm{oC}$ ODHsave.Icd

Method Filename : $5 \% \mathrm{iPrOH} 1 \overline{\mathrm{ml}} 20^{\circ} \mathrm{C}-\mathrm{OD}-\mathrm{H} . \mathrm{Icm}$

Batch Filename

Vial \#

Injection Volume

Date Acquired

Date Processed

50

29-6-2015 15:09-56

5-6-2015 15:09:56

Sample Type : Unknown

Acquired by : : System Administrator

Processed by : : System Administrator

\section{$<$ Chromatogram>}

mAU

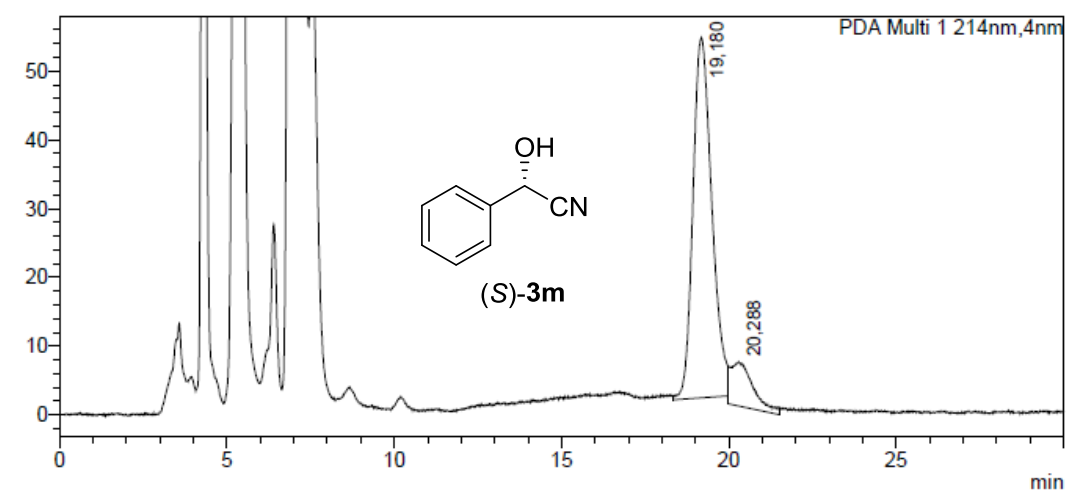

<Peak Table>

PDA Ch1 214nm
\begin{tabular}{|r|r|r|}
\hline Ret. Time & Area & \multicolumn{1}{|c|}{ Area $\%$} \\
\hline 19,180 & 2055313 & 86,902 \\
\hline 20,288 & 309769 & 13,098 \\
\hline & 2365082 & 100,000 \\
\hline
\end{tabular}




\section{References}

(1). De Mico, A.; Margarita, R.; Parlanti, L.; Vescovi, A.; Piancatelli, G. J. Org. Chem. 1997, 62 (20), 6974.

(2). (a) Beaufils, F.; Dénès, F.; Becattini, B.; Renaud, P.; Schenk, K. Adv. Synth. Catal. 2005, 347 (11-13), 1587. (b) More, J. D.; Finney, N. S. Org. Lett. 2002, 4 (17), 3001.

(3). Cioc, R. C.; Preschel, H. D.; van der Heijden, G.; Ruijter, E.; Orru, R. V. A Chem. - Eur. J. 2016, 22 , 7837.

(4). Watahiki, T.; Ohba, S.; Oriyama, T. Org. Lett. 2003, 5 (15), 2679. 